\title{
N-FOLD ČECH DERIVED FUNCTORS AND GENERALISED HOPF TYPE FORMULAS
}

\section{Guram Donadze}

A.Razmadze Mathematical Institute, Georgian Academy of Sciences

M.Alexidze St. 1, Tbilisi 380093. Georgia

donad@rmi.acnet.ge

Nick Inassaridze

A.Razmadze Mathematical Institute, Georgian Academy of Sciences

M.Alexidze St. 1, Tbilisi 380093. Georgia

inas@rmi.acnet.ge

\section{Timothy Porter}

Mathematics Division, School of Informatics, University of Wales Bangor,

Bangor, Gwynedd LL57 1UT, United Kingdom.

t.porter@bangor.ac.uk

\begin{abstract}
In 1988, Brown and Ellis published [3] a generalised Hopf formula for the higher homology of a group. Although substantially correct, their result lacks one necessary condition. We give here a counterexample to the result without that condition. The main aim of this paper is, however, to generalise this corrected result to derive formulae of Hopf type for the $n$-fold Čech derived functors of the lower central series functors $Z_{k}$. The paper ends with an application to algebraic K-theory.
\end{abstract}

\section{INTRODUCTION AND SUMMARY}

The well known Hopf formula for the second integral homology of a group says that for a given group $G$ there is an isomorphism

$$
H_{2}(G) \cong \frac{R \cap[F, F]}{[F, R]}
$$

where $R \nrightarrow F \rightarrow G$ is a free presentation of the group $G$.

Several alternative generalisations of this classical Hopf formula to higher dimensions were made in various papers, $[9,28,30]$, but perhaps the most successful one, giving formulas in all dimensions, was by Brown and Ellis, [3]. They used topological methods, and in particular the Hurewicz theorem for $n$-cubes of spaces, [5], which itself is an application of the generalised van Kampen theorem for diagrams of spaces [4]. The end result was:

1991 Mathematics Subject Classification. 18G50,18G10.

Key words and phrases. Cech derived functors, Hopf type formulas, $K$-functors. 
Theorem BE ([3]). Let $R_{1}, \ldots, R_{n}$ be normal subgroups of a group $F$ such that

$$
H_{2}(F)=0, \quad H_{r}\left(F / \prod_{i \in A} R_{i}\right)=0, \quad \text { for } r=|A|+1, r=|A|+2,
$$

with $A$ a non-empty proper subset of $\langle n\rangle=\{1, \ldots, n\}$ (for example, if the groups $F / \prod_{i \in A} R_{i}$ are free for $\left.A \neq\langle n\rangle\right)$ and $F / \prod_{1 \leq i \leq n} R_{i} \cong G$. Then there is an isomorphism

$$
H_{n+1}(G) \cong \frac{\bigcap_{i=1}^{n} R_{i} \cap[F, F]}{\prod_{A \subseteq\langle n\rangle}\left[\bigcap_{i \in A} R_{i}, \bigcap_{i \notin A} R_{i}\right]} .
$$

Later, Ellis using mainly algebraic means, and, in particular, his hyper-relative derived functors, proved the same result, [10].

The similarity between this formula and the formulae given by Mutlu and the third author for the various homotopy invariants of a simplicial group (see [19, 20]) suggested that there should be a purely algebraic proof of this, which hopefully would generalise further. Examining the classical case $(n=1)$, and the proof of the usual Hopf formula, showed a link with the Čech derived functors of the abelianisation functor, (cf. [12]).

Trying to derive this result purely algebraically and to obtain Hopf type formulas for some more general situations, we suspected that the conditions given above for Theorem BE were not sufficient for getting the generalised Hopf formula for $H_{n+1}(G), n \geq 3$. In fact, we give the following counter-example to Theorem BE:

Let $F$ be a free group with base $\left\{x_{1}, x_{2}\right\}, R_{1}, R_{2}$ and $R_{3}$ normal subgroups of the group $F$ generated by the one point sets $\left\{x_{1}\right\},\left\{x_{2}\right\}$ and $\left\{x_{1} x_{2}^{-1}\right\}$ respectively and $G=1$. Then we have $F / R_{i} \cong \mathbb{Z}, i=1,2,3, F / R_{i} R_{j}=1, i \neq j$ and $[F, F]=$ $\left[R_{1}, R_{2}\right]=R_{i} \cap R_{j}, i \neq j$, therefore

$$
\frac{\bigcap_{i \in\langle 3\rangle} R_{i} \cap[F, F]}{\prod_{A \subseteq\langle 3\rangle}\left[\bigcap_{i \in A} R_{i}, \bigcap_{i \notin A} R_{i}\right]} \cong \mathbb{Z}
$$

whilst $H_{3}(G)=1$.

We thus set out to prove a corrected version of this Brown-Ellis generalised Hopf formulae, but also to generalise it further in the following direction. Homology groups are the derived functors of the abelianization functor. Our generalisation handles the derived functors of the functors that kill higher commutators. More precisely, let the endofunctors $Z_{k}(G)$ be given by $Z_{k}(G)=G / \Gamma_{k}(G), k \geq 2$, where $\left\{\Gamma_{k}(G), k \geq 1\right\}$ is the lower central series of a given group $G$. These $Z_{k}$ are endofunctors on the category of groups and generalise the abelianization functor, so their non-abelian left derived functors, $L_{n} Z_{k}, n \geq 0$, generalise the group homology functors $H_{n}, n \geq 1$, cf., for instance, [1].

In [13], a Hopf-like formula is proved for the second Conduché-Ellis homology of precrossed modules using Čech derived functors. The main goal of this paper is to develop this method further, and by applying it, to express $L_{n} Z_{k}, n \geq 1, k \geq 2$, by 
generalised Hopf type formulas. In particular, we will give the stronger conditions needed for Theorem BE. Finally we apply these results to algebraic $K$-theory.

In the first section, we introduce the Cech derived functors illustrating their use by proving the classical Hopf formula in a new way. This is not just an illustration as it does indicate some of the ways the argument will go later on.

In Section 2 we introduce the notion of simple normal $(n+1)$-ad of groups $\left(F ; R_{1}, \ldots, R_{n}\right)$ relative to $R_{j}$ for some $1 \leq j \leq n$. Then we show that for a given pseudosimplicial group $F_{*}$, the normal $(j+1)$-ads of groups $\left(F_{n} ; \operatorname{Ker} d_{0}^{n}, \ldots, \operatorname{Ker} d_{j-1}^{n}\right)$ are simple relative to $\operatorname{Ker} d_{j-1}^{n}$ for all $1 \leq j \leq n$ (Proposition 7). The main result of Section 2 is Theorem 9, giving that for an aspherical augmented pseudosimplicial group $\left(F_{*}, d_{0}^{0}, G\right)$, there is a natural isomorphism

$$
\pi_{n} Z_{k}\left(F_{*}\right) \cong \frac{\bigcap_{i=0}^{n-1} \operatorname{Ker} d_{i}^{n-1} \cap \Gamma_{k}\left(F_{n-1}\right)}{D_{k}\left(F_{n-1} ; \operatorname{Ker} d_{0}^{n-1}, \ldots, \operatorname{Ker} d_{n-1}^{n-1}\right)}
$$

for $n \geq 1, k \geq 2$. Here the $D_{k}$-term takes the form of an iterated commutator subgroup which is an obvious generalisation of the denominator terms of both the classical Hopf formula and the Brown-Ellis extension of that formula. It is also related to the descriptions of the image term of the Moore complex, as used in $[19,20]$. The explicit formula is given at the start of Section 2.

For an inclusion crossed $n$-cube of groups, $\mathcal{M}$, given by a normal $(n+1)$-ad of groups we construct, in Section 3, a new induced crossed $n$-cube $\mathcal{B}_{k}(\mathcal{M}), k \geq 2$ (Proposition 11). We show the existence of an isomorphism of simplicial groups $Z_{k} E^{(n)}(\mathcal{M})_{*} \cong E^{(n)}\left(\mathcal{B}_{k}(\mathcal{M})\right)_{*}$, where $E^{(n)}(\mathcal{M})_{*}$ denotes the diagonal of the $n$ simplicial nerve of the crossed $n$-cube of groups $\mathcal{M}$, (Proposition 12).

Section 4 is devoted to the investigation of some properties of the mapping cone complex of a morphism of (non-abelian) group complexes introduced in [17]. In particular, for a given morphism of pseudosimplicial groups $\alpha: G_{*} \rightarrow H_{*}$ the natural morphism $\kappa: N M_{*}(\alpha) \rightarrow C_{*}(\widetilde{\alpha})$ induces isomorphisms of their homology groups, where $C_{*}(\widetilde{\alpha})$ is the mapping cone complex of the induced morphism of the Moore complexes and $N M_{*}(\alpha)$ is the Moore complex of a new pseudosimplicial group constructed using $\alpha$ (Proposition 13). (Here similar results have recently been found by Conduché, [8].) Using this result we derive purely algebraicly the result of [17], (3.4. Proposition), giving for a crossed $n$-cube of groups $\mathcal{M}$ an isomorphism between the homotopy groups of $E^{(n)}(\mathcal{M})_{*}$ and the corresponding homology groups of a chain complex of groups $C_{*}(\mathcal{M})$, (Proposition 14). In particular, we give an explicit computation of the $n$-th homotopy group of the $\operatorname{simplicial}$ group $E^{(n)}(\mathcal{M})_{*}$.

In Section 5, we introduce a notion of $n$-fold Čech derived functors of an endofunctor on the category of groups (Theorem 16, Definition) which will be the subject of future papers and applications to nonabelian homological algebra and $K$-theory. We give an explicit calculation of $n$-th $n$-fold Čech derived functor of the functor $Z_{k}, k \geq 2$ (Theorem 20). Our method gives the possibility of finding the sought for sufficient conditions for, and a purely algebraic proof of, the generalised Hopf 
formula of Brown and Ellis, moreover we express $L_{n} Z_{k}(G), n \geq 1, k \geq 2$ by a Hopf type formula (Theorem 21).

In Section 6, an application to algebraic $K$-theory is given. In particular, Quillen's algebraic $K$-functors $K_{n+1}, n \geq 1$ are described in terms of short exact sequence including the higher Hopf type formulae for free exact $n$-presentations induced by a free simplicial resolution of the general linear group (Theorem 25).

\section{An approach to the Classical Hopf formula via Čech Derived FUNCTORS}

We give here a brief introduction to Čech derived functors. A fuller account is given in [12]. We will limit ourselves to the Cech derived functors of the Abelianization functor. Later we will develop the $n$-fold analogue of some of this theory.

Definition. Let $T: \mathfrak{G} r \rightarrow \mathfrak{G} r$ be a covariant functor. Define $i$-th $\check{C}$ ech derived functor $\mathcal{L}_{i} T: \mathfrak{G} r \rightarrow \mathfrak{G} r, i \geq 0$, of the functor $T$ by choosing for each $G$ in $\mathfrak{G} r$, a free presentation $\mathfrak{F}: R \longmapsto F \stackrel{\alpha}{\rightarrow} G$ of $G$ and setting

$$
\mathcal{L}_{i} T(G)=\pi_{i}\left(T \check{C}(\alpha)_{*}\right)
$$

where $\left(\check{C}(\alpha)_{*}, \alpha, G\right)$ is the Čech resolution of the group $G$ for the free presentation $\mathfrak{F}$ of $G$. This latter resolution is constructed as follows:

Given a group $G$ and a homomorphism of groups $\alpha: F \rightarrow G$. The $\check{C}$ ech augmented complex $\left(\check{C}(\alpha)_{*}, \alpha, G\right)$ for $\alpha$ is

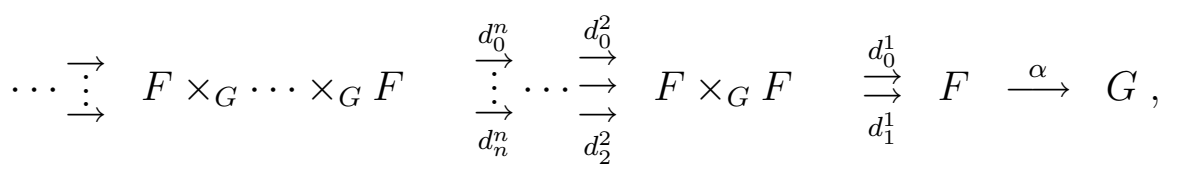

thus

$$
\begin{gathered}
\check{C}(\alpha)_{n}=\underbrace{F \times_{G} \cdots \times_{G} F}_{(n+1) \text {-times }}=\left\{\left(x_{0}, \ldots, x_{n}\right) \in F^{n+1} \mid \alpha\left(x_{0}\right)=\cdots=\alpha\left(x_{n}\right)\right\} \text { for } n \geq 0, \\
d_{i}^{n}\left(x_{0}, \ldots, x_{n}\right)=\left(x_{0}, \ldots, \hat{x}_{i}, \ldots, x_{n}\right)
\end{gathered}
$$

and

$$
s_{i}^{n}\left(x_{0}, \ldots, x_{n}\right)=\left(x_{0}, \ldots, x_{i}, x_{i}, x_{i+1}, \ldots, x_{n}\right)
$$

for $0 \leq i \leq n$ (see [12]).

In case $F$ is a free group and $\alpha$ is an epimorphism as in $\mathfrak{F}$ above, $\left(\check{C}(\alpha)_{*}, \alpha, G\right)$ will be called a Čech resolution of $G$. Example. The prime example of the Čech derived functors are those of the abelianization functor. We recall that

$$
H_{2}(G) \cong \mathcal{L}_{1} A b(G)
$$

and will use this later.

Now using crossed modules and their nerves, we present a fresh view of the Cech complex which leads to some ideas that will be useful throughout the paper. 
First, let us recall the definition of crossed module. A crossed module $(M, P, \mu)$ is a group homomorphism $\mu: M \rightarrow P$ together with a (left) action of $P$ on $M$ which satisfies the following conditions:

(i) $\mu\left({ }^{p} m\right)=p \mu(m) p^{-1}$,

(ii) ${ }^{\mu(m)} m^{\prime}=m m^{\prime} m^{-1}, \quad$ (Peiffer identity)

for all $m, m^{\prime} \in M$ and $p \in P$.

A morphism $(\varphi, \psi):(M, P, \mu) \rightarrow(N, Q, \nu)$ of crossed modules is a commutative square of groups



such that $\varphi\left({ }^{p} m\right)={ }^{\psi(p)} \varphi(m)$ for all $m \in M, p \in P$. Let us denote the category of crossed modules by $\mathcal{C} \mathcal{M}$.

It is well known from [17] that the category $\mathcal{C} M$ of crossed modules is equivalent to the category of $c a t^{1}$-groups (for the definition see [17]), and for a given crossed module, $\mathcal{M}=(M \stackrel{\mu}{\rightarrow} P)$, the corresponding cat ${ }^{1}$-group is $(M \rtimes P, s, t)$, where $s(m, p)=p$ and $t(m, p)=\mu(m) p$. This $c a t^{1}$-group has an internal category stucture within the category $\mathfrak{G} \mathfrak{r}$ of groups and the nerve of its category structure forms the following simplicial group

$$
E(\mathcal{M})_{*}: \quad \cdots \underset{\vdots}{\longrightarrow} E(\mathcal{M})_{n} \underset{\vdots}{\longrightarrow} \quad \cdots \quad \stackrel{\stackrel{d_{0}}{\rightleftharpoons}}{\underset{d_{2}}{\rightleftarrows}} E(\mathcal{M})_{1} \stackrel{d_{0}}{\underset{d_{1}}{\longrightarrow}} E(\mathcal{M})_{0}
$$

where $E(\mathcal{M})_{n}=M \rtimes(\cdots(M \rtimes P) \cdots)$ with $n$ semidirect factors of $M$, and face and degeneracy homomorphisms are defined by

$$
\begin{aligned}
& d_{0}\left(m_{1}, \ldots, m_{n}, p\right)=\left(m_{2}, \ldots, m_{n}, p\right) \\
& d_{i}\left(m_{1}, \ldots, m_{n}, p\right)=\left(m_{1}, \ldots, m_{i} m_{i+1}, \ldots, m_{n}, p\right), 0<i<n, \\
& d_{n}\left(m_{1}, \ldots, m_{n}, p\right)=\left(m_{1}, \ldots, m_{n-1}, \mu\left(m_{n}\right) p\right), \\
& s_{i}\left(m_{1}, \ldots, m_{n}, p\right)=\left(m_{1}, \ldots, m_{i}, 1, m_{i+1}, \ldots, m_{n}, p\right), \quad 0 \leq i \leq n .
\end{aligned}
$$

The simplicial group $E(\mathcal{M})_{*}$ is called the nerve of the crossed module $\mathcal{M}$ and its Moore complex is trivial in dimensions $\geq 2$. In fact its Moore complex is just the original crossed module up to isomorphism with $M$ in dimension 1 and $P$ in dimension 0 .

Lemma 1. Let $G$ be a group and $F \stackrel{\alpha}{\rightarrow} G$ be a homomorphism of groups. Then the Cech complex for $\alpha$ is isomorphic to the nerve of the inclusion crossed module $E(R \hookrightarrow F)_{*}$, where $R=\operatorname{Ker} \alpha$. 
Proof. Let us compare the following two simplicial groups, the Čech complex for $\alpha$ and the nerve of the inclusion crossed module $R \hookrightarrow F$

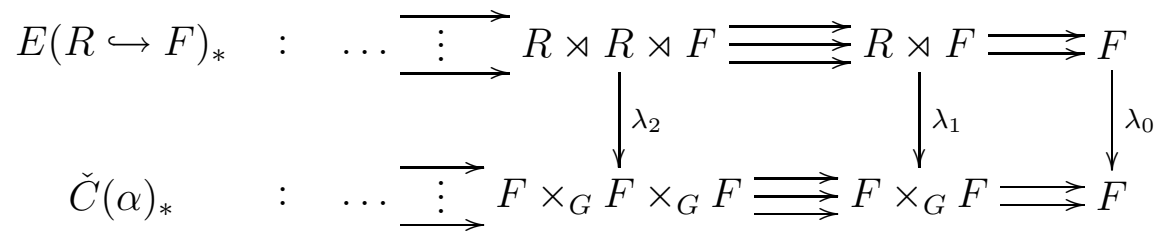

by constructing a morphism $\lambda_{*}$ as follows:

$\lambda_{0}$ is the identity on $F$; and

$\lambda_{n}\left(r_{1}, \ldots, r_{n}, f\right)=\left(r_{1} \cdots r_{n} f, r_{2} \cdots r_{n} f, \ldots, r_{n} f, f\right)$ for all $n \geq 1$ and $\left(r_{1}, \ldots, r_{n}, f\right) \in E(R \hookrightarrow F)_{n}$.

It is easy to check that $\lambda_{*}$ is isomorphism of simplicial groups.

We recall from [16] that a crossed module $\mu: M \rightarrow P$ is called abelian if $P$ is an abelian group and the action of $P$ on $M$ is trivial. This implies that $M$ is also abelian. Let us denote the category of abelian crossed modules by $\mathfrak{A} b \mathcal{C} M$.

One can define the abelianization functor $A b$ from the category $\mathcal{C} M$ to the category $\mathfrak{A} b \mathcal{C} M$ in the following way: for any crossed module $\mathcal{M}=(M \stackrel{\mu}{\rightarrow} P)$,

$$
A b(\mathcal{M})=\left(\frac{M}{[P, M]} \stackrel{\bar{\mu}}{\rightarrow} \frac{P}{[P, P]}\right),
$$

in which $[P, M]$ is the subgroup of $M$ generated by the elements ${ }^{p} m^{-1}$ for all $m \in M, p \in P$ and $\bar{\mu}$ is induced by $\mu$.

Given a simplicial group $G_{*}$, let us apply the group abelianization functor dimensionwise, denote the resulting simplicial group by $A b\left(G_{*}\right)$.

Proposition 2. Let $M \stackrel{\mu}{\rightarrow} P$ be a crossed module. Then there is an isomorphism of simplicial groups

$$
A b\left(E(M \stackrel{\mu}{\rightarrow} P)_{*}\right) \cong E(A b(M \stackrel{\mu}{\rightarrow} P))_{*} .
$$

Proof. Let us consider the two simplicial groups

$$
A b\left(E(M \stackrel{\mu}{\rightarrow} P)_{*}\right): \quad: \quad \ldots \quad \stackrel{\square}{\longrightarrow}(M \rtimes M \rtimes P)^{a b} \rightrightarrows(M \rtimes P)^{a b} \rightrightarrows P^{a b}
$$

and

$E(A b(M \stackrel{\mu}{\rightarrow} P))_{*}: \quad: \quad \ldots \underset{\longrightarrow}{\longrightarrow} \frac{M}{[P, M]} \times \frac{M}{[P, M]} \times P^{a b} \Longrightarrow \frac{M}{\Longrightarrow} \times P^{a b} \Longrightarrow P^{a b}$.

It is easy to show that

and

$$
\begin{gathered}
(M \rtimes P)^{a b} \stackrel{\kappa}{\rightarrow} \frac{M}{[P, M]} \times P^{a b}, \\
\kappa[(m, p)]=([m],[p])
\end{gathered}
$$

$$
\frac{M}{[P, M]} \times P^{a b} \stackrel{\kappa^{\prime}}{\rightarrow}(M \rtimes P)^{a b},
$$




$$
\kappa^{\prime}([m],[p])=[(m, p)]
$$

are homomorphisms and $\kappa \kappa^{\prime}=\kappa^{\prime} \kappa=1$. Using these isomorphisms one has

$$
\begin{aligned}
(M \rtimes(M \rtimes P))^{a b} & \cong \frac{M}{[M \rtimes P, M]} \times(M \rtimes P)^{a b} \\
& \cong \frac{M}{[M \rtimes P, M]} \times \frac{M}{[P, M]} \times P^{a b} \\
& \cong \frac{M}{[P, M]} \times \frac{M}{[P, M]} \times P^{a b}
\end{aligned}
$$

since $[M \rtimes P, M]=[P, M]$ as

$$
\begin{aligned}
{ }^{(m, p)} m^{\prime} m^{\prime-1} & =m^{p} m^{\prime} m^{-1} m^{\prime-1} \\
& =m^{p} m^{\prime} m^{\prime-1} m^{-1} m m^{\prime} m^{-1} m^{\prime-1} \\
& =m\left({ }^{p} m^{\prime} m^{\prime-1}\right) m^{-1}\left({ }^{\mu(m)} m^{\prime} m^{\prime-1}\right) \in[P, M]
\end{aligned}
$$

for all $m, m^{\prime} \in M, p \in P$. It is also easy to see by the same sort of argument that there exist isomorphisms between higher terms of these simplicial groups and that these isomorphisms are compatible with face and degeneracy maps.

Now we are ready to revisit the classical Hopf formula.

One can prove the Hopf formula in many ways, but for our later generalization we will prove it using the Čech derived functors.

Theorem 3. Let $G$ be a group and $R \stackrel{i}{\longmapsto} F \rightarrow G$ be a free presentation of the group $G$. Then there is an isomorphism of groups

$$
H_{2}(G) \cong \frac{R \cap[F, F]}{[F, R]} .
$$

Proof. Using [22, 24] (see also Theorem 2.39(ii), [12]), there is an isomorphism

$$
H_{2}(G) \cong \mathcal{L}_{1} A b(G)
$$

where $\mathcal{L}_{1} A b$ is the first Cech derived functor of the group abelianization functor.

Now Lemma 1 and Proposition 2 implies an isomorphism

$$
H_{2}(G) \cong \pi_{1}\left(E(A b(R \stackrel{i}{\hookrightarrow} F))_{*}\right) .
$$

As we already mentioned above, it is clear that $\pi_{1}\left(E(A b(R \hookrightarrow F))_{*}\right)$ is isomorphic to the kernel of the crossed module

$$
A b(R \hookrightarrow F)=\left(\frac{R}{[F, R]} \stackrel{\bar{i}}{\longrightarrow} \frac{F}{[F, F]}\right)
$$

giving the desired result. 
Corollary 4. Let $R \longmapsto K \stackrel{\alpha}{\rightarrow} G$ be a presentation of a group $G$ and $H_{2}(K)=0$. Then there is an isomorphism of groups

$$
H_{2}(G) \cong \frac{R \cap[K, K]}{[K, R]} .
$$

Proof. Consider a free presentation $R^{\prime \prime} \longmapsto F \stackrel{\beta}{\rightarrow} K$ of the group $K$. Hence, one has also a free presentation $R^{\prime} \longmapsto F \stackrel{\alpha \beta}{\rightarrow} G$ of the group $G$. It is easy to check that there is an exact sequence of groups

$$
\frac{R^{\prime \prime} \cap[F, F]}{\left[F, R^{\prime \prime}\right]} \longrightarrow \frac{R^{\prime} \cap[F, F]}{\left[F, R^{\prime}\right]} \longrightarrow \frac{R}{[K, R]} \longrightarrow \frac{K}{[K, K]} \longrightarrow \frac{G}{[G, G]} \longrightarrow 1,
$$

Thus by Theorem 3 and the condition that $H_{2}(K)=0$, one has the following exact sequence of groups

$$
0 \longrightarrow H_{2}(G) \longrightarrow \frac{R}{[K, R]} \longrightarrow K^{a b} \longrightarrow G^{a b} \longrightarrow 1
$$

which completes the proof.

\section{Simple NORMAL $(n+1)$-AD OF GROUPS}

One of the tools we will be using later is the theory of crossed $n$-cubes of groups. These generalise normal $(n+1)$-ads of groups in the same way that crossed modules generalise normal subgroups. We therefore start by developing some techniques for handling $(n+1)$-ads of groups, relating them to iterated commutators.

Given a group $F$ and $n$ normal subgroups, $R_{1}, \ldots, R_{n}$, then $\left(F ; R_{1}, \ldots, R_{n}\right)$ will be called a normal $(n+1)$-ad of groups. A normal $(n+1)$-ad of groups $\left(F ; R_{1}, \ldots, R_{n}\right)$ is called simple relative to $R_{j}$ for some $1 \leq j \leq n$ if there exists a subgroup $F^{\prime}$ of the group $F$ such that

$$
F^{\prime} \cap R_{j}=1, \quad \bigcap_{i \in A} R_{i}=\left(\cap_{i \in A} R_{i} \cap F^{\prime}\right)\left(\cap_{i \in A} R_{i} \cap R_{j}\right)
$$

for all $A \subseteq\langle n\rangle \backslash\{j\}$.

For a given $(n+1)$-ad of groups $\left(F ; R_{1}, \ldots, R_{n}\right), A \subseteq\langle n\rangle$ and $k \geq 1$ denote by $D_{k}(F ; A)$ the following normal subgroup of the group $F$

$$
\prod_{A_{1} \cup A_{2} \cup \ldots \cup A_{k}=A}\left[\bigcap_{i \in A_{1}} R_{i},\left[\underset{i \in A_{2}}{\cap} R_{i}, \ldots,\left[\underset{i \in A_{k-1}}{\cap} R_{i}, \underset{i \in A_{k}}{\cap} R_{i}\right] \ldots\right]\right] .
$$

Sometimes we write $D_{k}\left(F ; R_{1}, \ldots, R_{n}\right)$ instead of the notation $D_{k}(F ;\langle n\rangle)$.

Lemma 5. Let $\left(F ; R_{1}, \ldots, R_{n}\right)$ be a normal $(n+1)$-ad of groups which is simple relative to $R_{j}, 1 \leq j \leq n$ and $k \geq 1$, then

$$
D_{k}(F ; A)=\left(D_{k}(F ; A) \cap F^{\prime}\right) D_{k}(F ; A \cup\{j\})
$$

for all $A \subseteq\langle n\rangle \backslash\{j\}$. 
Proof. We use induction on $k$. Let $k=1$, then

$$
D_{1}(F ; A)=\bigcap_{i \in A} R_{i}=\left(\cap_{i \in A} R_{i} \cap F^{\prime}\right)\left(\cap_{i \in A} R_{i} \cap R_{j}\right)=\left(\cap_{i \in A} R_{i} \cap F^{\prime}\right) D_{1}(F ; A \cup\{j\})
$$

for $A \subseteq\langle n\rangle \backslash\{j\}$.

Proceeding by induction, we suppose that the assertion is true for $k-1$ and we will prove it for $k$.

The inclusion $\left(D_{k}(F ; A) \cap F^{\prime}\right) D_{k}(F ; A \cup\{j\}) \subseteq D_{k}(F ; A)$ is obvious. It is easy to see that a generator of $D_{k}(F ; A)$ has the form $[x, w]$, where $x \in \bigcap_{i \in B} R_{i}, w \in$ $D_{k-1}(F ; C), B, C \subseteq A \subseteq\langle n\rangle \backslash\{j\}$ and $B \cup C=A$. There exist elements $y \in$ $\bigcap_{i \in B} R_{i} \cap F^{\prime}$ and $z \in \cap_{i \in B} R_{i} \cap R_{j}$ such that $x=y z$. One has

$$
[x, w]=[y z, w]=y[z, w] y^{-1}[y, w] .
$$

Clearly $[z, w] \in D_{k}(F ; B \cup C \cup\{j\})=D_{k}(F ; A \cup\{j\})$ and hence $y[z, w] y^{-1} \in$ $D_{k}(F ; A \cup\{j\})$. By inductive hypothesis there exist $w^{\prime} \in D_{k-1}(F ; C \cup\{j\})$ and $x^{\prime} \in D_{k-1}(F ; C) \cap F^{\prime}$ such that $w=x^{\prime} w^{\prime}$. One has

$$
[y, w]=\left[y, x^{\prime} w^{\prime}\right]=\left[y, x^{\prime}\right] x^{\prime}\left[y, w^{\prime}\right] x^{\prime-1} .
$$

Clearly $\left[y, w^{\prime}\right] \in D_{k}(F ; B \cup C \cup\{j\})=D_{k}(F ; A \cup\{j\})$ and hence $x^{\prime}\left[y, w^{\prime}\right] x^{\prime-1} \in$ $D_{k}(F ; A \cup\{j\})$. Therefore there is an element $w^{\prime \prime} \in D_{k}(F ; A \cup\{j\})$ such that $[x, w]=\left[y, x^{\prime}\right] w^{\prime \prime}$ where $\left[y, x^{\prime}\right] \in D_{k}(F ; A) \cap F^{\prime}$.

For a given group $G$ the (lower) central series $\Gamma_{k}=\Gamma_{k}(G)$,

$$
G=\Gamma_{1} \supseteq \Gamma_{2} \supseteq \cdots \supseteq \Gamma_{k} \supseteq \cdots
$$

of $G$ is defined inductively by $\Gamma_{k}=\prod_{i+j=k}\left[\Gamma_{i}, \Gamma_{j}\right]$. The well-known Witt-Hall identities on commutators (see e.g. [2]) imply that $\Gamma_{k}=\left[G, \Gamma_{k-1}\right]$.

Let $\mathfrak{G} r$ denote the category of groups. Let us define higher abelianization type functors $Z_{k}: \mathfrak{G} r \rightarrow \mathfrak{G} r, k \geq 2$ by $Z_{k}(G)=G / \Gamma_{k}(G)$ for any $G \in$ ob $\mathfrak{G} r$ and where $Z_{k}(\alpha)$ is the natural homomorphism induced by a group homomorphism $\alpha$. Of course, $Z_{2}$ is the ordinary abelianization functor of groups.

Proposition 6. Let $\left(F ; R_{1}, \ldots, R_{n}\right)$ be a normal $(n+1)$-ad of groups and $k \geq 2$. Suppose that $\left(F ; R_{1}, \ldots, R_{j}\right)$ is a simple normal $(j+1)$-ad of groups relative to $R_{j}$ for all $1 \leq j \leq n$. Then

$$
\bigcap_{i \in\langle j\rangle} R_{i} \cap \Gamma_{k}(F)=D_{k}(F ;\langle j\rangle), \quad 1 \leq j \leq n
$$

Proof. Since the inclusion $D_{k}(F ;\langle j\rangle) \subseteq \bigcap_{i \in\langle j\rangle} R_{i} \cap \Gamma_{k}(F)$ is clear, we only have to show the inclusion $\underset{i \in\langle j\rangle}{\cap} R_{i} \cap \Gamma_{k}(F) \subseteq D_{k}(F ;\langle j\rangle)$, which will be done by induction on $j$.

Let $j=1$, then there exists a subgroup $F_{1}$ of the group $F$ such that $R_{1} \cap F_{1}=1$ and $F=F_{1} R_{1}$. Let $w \in R_{1} \cap \Gamma_{k}(F) \subseteq \Gamma_{k}(F)=D_{k}(F ; \emptyset)$. Using Lemma 5 one has elements $x^{\prime} \in D_{k}(F ; \emptyset) \cap F_{1}$ and $w^{\prime} \in D_{k}(F ;\langle 1\rangle)$ such that $w=x^{\prime} w^{\prime}$. But $x^{\prime}=w w^{\prime-1} \in R_{1}$ and hence $x^{\prime}=1$. Thus $R_{1} \cap \Gamma_{k}(F) \subseteq D_{k}(F ;\langle 1\rangle)$. 
Proceeding by induction, we suppose that the result is true for $j-1$ and we will prove it for $j$.

There exists a subgroup $F_{j}$ of the group $F$ such that $R_{j} \cap F_{j}=1$ and $\cap_{i \in A} R_{i}=$ $\left(\cap_{i \in A} R_{i} \cap F_{j}\right)\left(\cap_{i \in A} R_{i} \cap R_{j}\right)$ for all $A \subseteq\{1, \ldots, j-1\}$. Let $w \in \underset{i \in\langle j\rangle}{\cap} R_{i} \cap \Gamma_{k}(F) \subseteq$ $\bigcap_{i \in\langle j-1\rangle} R_{i} \cap \Gamma_{k}(F)$. Using the inductive hypothesis one has the equality $\bigcap_{i \in\langle j-1\rangle} R_{i} \cap$ $\Gamma_{k}(F)=D_{k}(F ;\langle j-1\rangle)$. By Lemma 5 there are elements $x^{\prime} \in D_{k}(F ;\langle j-1\rangle) \cap F_{j}$ and $w^{\prime} \in D_{k}(F ;\langle j\rangle)$ such that $w=x^{\prime} w^{\prime}$. Certainly $x^{\prime}=w w^{\prime}-1 \in R_{j}$ and hence $x^{\prime}=1$. Therefore $\bigcap_{i \in\langle j\rangle} R_{i} \cap \Gamma_{k}(F) \subseteq D_{k}(F ;\langle j\rangle)$.

These conditions may seem rather restrictive, but the following observation shows that examples of simple normal $(n+1)$-ads of groups appear naturally, and that moreover these examples satisfy the conditions of Proposition 6. First some terminology and notation on pseudosimplicial groups, (cf. [12] for the general theory).

A pseudosimplicial group $G_{*}$ is a non-negatively graded group with face homomorphisms $d_{i}^{n}: G_{n} \rightarrow G_{n-1}$ and pseudodegeneracies, $s_{i}^{n}: G_{n} \rightarrow G_{n+1}, 0 \leq i \leq n$, satisfying all the simplicial identities except possibly the identity $s_{i}^{n+1} s_{j}^{n}=s_{j+1}^{n+1} s_{i}^{n}$ for $i \leq j$ (again, see [12]). The Moore complex $\left(N G_{*}, \partial_{*}\right)$ of $G_{*}$ is the chain complex defined by $N G_{n}=\bigcap_{i=0}^{n-1} \operatorname{Ker} d_{i}^{n}$ with $\partial_{n}: N G_{n} \rightarrow N G_{n-1}$ induced from $d_{n}^{n}$ by restriction. The homotopy groups of $G_{*}$ are defined as the homology groups of the complex $\left(N G_{*}, \partial_{*}\right)$, i.e. $\pi_{n}\left(G_{*}\right)=H_{n}\left(N G_{*}, \partial_{*}\right), n \geq 0$ (see [12]). For good examples of pseudosimplicial groups see [18].

Proposition 7. Let $F_{*}$ be a pseudosimplicial group. Then $\left(F_{n} ; \operatorname{Ker} d_{0}^{n}, \ldots, \operatorname{Ker} d_{j-1}^{n}\right)$ is a simple normal $(j+1)$-ad of groups relative to $\operatorname{Ker} d_{j-1}^{n}$ for all $1 \leq j \leq n$.

Proof. Since $d_{j-1}^{n} s_{j-1}^{n-1}=1, s_{j-1}^{n-1}\left(F_{n-1}\right) \cap \operatorname{Ker} d_{j-1}^{n}=1$ and $s_{j-1}^{n-1}\left(F_{n-1}\right) \operatorname{Ker} d_{j-1}^{n}=F_{n}$ for all $n \geq 1$. Hence when $j=1,\left(F_{n}\right.$; Ker $\left.d_{0}^{n}\right)$ is a simple normal 2-ad of groups relative to $\operatorname{Ker} d_{0}^{n}$ and the $F^{\prime}$ of the definition of simplicity is $s_{0}^{n-1}\left(F_{n-1}\right)$.

Now suppose that $j>1$. We will show the following equality

$$
\bigcap_{i \in A} \operatorname{Ker} d_{i}^{n}=\left(\cap_{i \in A} \operatorname{Ker} d_{i}^{n} \cap s_{j-1}^{n-1}\left(F_{n-1}\right)\right)\left(\cap_{i \in A} \operatorname{Ker} d_{i}^{n} \cap \operatorname{Ker} d_{j-1}^{n}\right)
$$

for all $A \subseteq\{0, \ldots, j-2\}$ and $A \neq \emptyset$, so again the $F^{\prime}$ of the definition of simplicity is $s_{j-1}^{n-1}\left(F_{n-1}\right)$. Let $x=s_{j-1}^{n-1}\left(x_{n-1}\right) r_{j-1} \in \bigcap_{i \in A} \operatorname{Ker} d_{i}^{n}$, where $x_{n-1} \in F_{n-1}, r_{j-1} \in$ $\operatorname{Ker} d_{j-1}^{n}$. Thus $d_{i}^{n}(x)=d_{i}^{n} s_{j-1}^{n-1}\left(x_{n-1}\right) d_{i}^{n}\left(r_{j-1}\right)=1$ for all $i \in A$. Since $i<j-1$, one has $d_{i}^{n}\left(r_{j-1}\right)=s_{j-2}^{n-2} d_{i}^{n-1}\left(x_{n-1}\right)^{-1}$. Hence $1=d_{i}^{n-1} d_{j-1}^{n}\left(r_{j-1}\right)=d_{j-2}^{n-1} d_{i}^{n}\left(r_{j-1}\right)=$ $d_{j-2}^{n-1} s_{j-2}^{n-2} d_{i}^{n-1}\left(x_{n-1}\right)^{-1}=d_{i}^{n-1}\left(x_{n-1}\right)^{-1}$. Therefore $d_{i}^{n}\left(r_{j-1}\right)=1$ and $d_{i}^{n} s_{j-1}^{n-1}\left(x_{n-1}\right)=$ 1 for all $i \in A$.

The next lemma is well known, but very useful. The proof is routine.

Lemma 8. Let $G_{*}$ be a pseudosimplicial group and $A \subseteq\langle n\rangle, A \neq\langle n\rangle$, then $d_{n}^{n}\left(\bigcap_{i \in A} \operatorname{Ker} d_{i-1}^{n}\right)=\bigcap_{i \in A} \operatorname{Ker} d_{i-1}^{n-1}, n \geq 2$. 
Next let us consider an augmented pseudosimplicial group $\left(F_{*}, d_{0}^{0}, G\right)$ and, applying the functor $Z_{k}$ dimension-wise, denote the resulting augmented pseudosimplicial group by $\left(Z_{k}\left(F_{*}\right), Z_{k}\left(d_{0}^{0}\right), Z_{k}(G)\right)$. Our previous results will allow calculation of the homotopy groups of $Z_{k}\left(F_{*}\right)$ in certain important cases notably the following.

Theorem 9. If $\left(F_{*}, d_{0}^{0}, G\right)$ is aspherical then there is a natural isomorphism

$$
\pi_{n} Z_{k}\left(F_{*}\right) \cong \frac{\bigcap_{i=0}^{n-1} \operatorname{Ker} d_{i}^{n-1} \cap \Gamma_{k}\left(F_{n-1}\right)}{D_{k}\left(F_{n-1} ; \operatorname{Ker} d_{0}^{n-1}, \ldots, \operatorname{Ker} d_{n-1}^{n-1}\right)}, \quad k \geq 2, n \geq 1 .
$$

Proof. Let us consider the short exact sequence of augmented pseudosimplicial groups

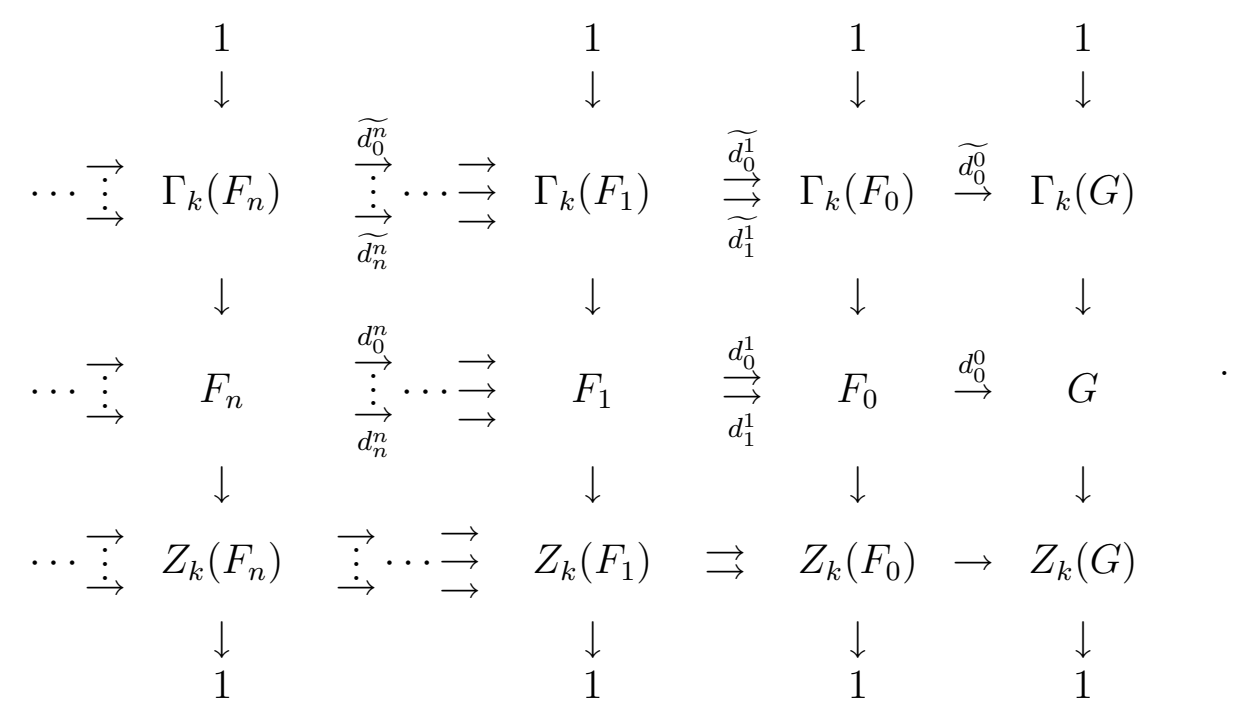

By the induced long exact homotopy sequence, one has the isomorphisms of groups

$$
\pi_{n} Z_{k}\left(F_{*}\right) \cong \frac{\bigcap_{i=0}^{n-1} \operatorname{Ker} \widetilde{d_{i}^{n-1}}}{\widetilde{d_{n}^{n}}\left(\bigcap_{i=0}^{n-1} \operatorname{Ker} \widetilde{d_{i}^{n}}\right)}, \quad n \geq 1 .
$$

Since $\widetilde{d_{i}^{n}}$ is a restriction of $d_{i}^{n}$ to $\Gamma_{k}\left(F_{n}\right)$, Ker $\widetilde{d_{i}^{n}}=\operatorname{Ker} d_{i}^{n} \cap \Gamma_{k}\left(F_{n}\right)$. Hence $\bigcap_{i=0}^{n-1} \operatorname{Ker} \widetilde{d_{i}^{n-1}}=\left(\bigcap_{i=0}^{n-1} \operatorname{Ker} d_{i}^{n-1}\right) \cap \Gamma_{k}\left(F_{n-1}\right)$ and $\bigcap_{i=0}^{n-1} \operatorname{Ker} \widetilde{d_{i}^{n}}=\left(\bigcap_{i=0}^{n-1} \operatorname{Ker} d_{i}^{n}\right) \cap \Gamma_{k}\left(F_{n}\right)$.

Using Proposition 6 and Proposition 7 one has

$$
\left(\cap_{i \in\langle n\rangle} \operatorname{Ker} d_{i-1}^{n}\right) \cap \Gamma_{k}\left(F_{n}\right)=D_{k}\left(F_{n} ; \operatorname{Ker} d_{0}^{n}, \ldots, \operatorname{Ker} d_{n-1}^{n}\right)
$$

for $n \geq 1$.

Since $\left(F_{*}, d_{0}^{0}, G\right)$ is an aspherical augmented pseudosimplicial group, $d_{n}^{n}\left(\underset{i \in\langle n\rangle}{\cap} \operatorname{Ker} d_{i-1}^{n}\right)=\bigcap_{i \in\langle n\rangle} \operatorname{Ker} d_{i-1}^{n-1}, n \geq 1$. Using this fact and Lemma 8, it is now easy to see that one has an equality

$\widetilde{d_{n}^{n}}\left(\bigcap_{i=0}^{n-1} \operatorname{Ker} \widetilde{d_{i}^{n}}\right)=d_{n}^{n}\left(D_{k}\left(F_{n} ; \operatorname{Ker} d_{0}^{n}, \ldots, \operatorname{Ker} d_{n-1}^{n}\right)\right)=D_{k}\left(F_{n-1} ; \operatorname{Ker} d_{0}^{n-1}, \ldots, \operatorname{Ker} d_{n-1}^{n-1}\right)$. 
In the case where $\left(F_{*}, d_{0}^{0}, G\right)$ is a free pseudosimplicial resolution of $G$, these homotopy groups will be the left non-abelian derived functors $L_{n} Z_{k}(G)$ of $Z_{k}$, evaluated at $G$. (For more on these non-abelian derived functors, we refer the reader to [12].) We thus have the following formal result.

Corollary 10. Let $G$ be a group and $\left(F_{*}, d_{0}^{0}, G\right)$ an aspherical augmented pseudosimplicial group and $k \geq 2$. If $F_{n}$ is a free group for all $n \geq 0$, i.e. $\left(F_{*}, d_{0}^{0}, G\right)$ is a free pseudosimplicial resolution of the group $G$, then there is a natural isomorphism

$$
L_{n} Z_{k}(G) \cong \frac{\bigcap_{i=0}^{n-1} \operatorname{Ker} d_{i}^{n-1} \cap \Gamma_{k}\left(F_{n-1}\right)}{D_{k}\left(F_{n-1} ; \operatorname{Ker} d_{0}^{n-1}, \ldots, \operatorname{Ker} d_{n-1}^{n-1}\right)}, \quad n \geq 1 .
$$

Proof. Straightforward from Theorem 9.

\section{Crossed $n$-Cubes of Groups}

The following definition is due to Ellis and Steiner [11] (see also [25]). A crossed $n$ cube of groups is a family $\left\{\mathcal{M}_{A}: A \subseteq\langle n\rangle\right\}$ of groups, together with homomorphisms, $\mu_{i}: \mathcal{M}_{A} \rightarrow \mathcal{M}_{A \backslash\{i\}}$ for $i \in\langle n\rangle, A \subseteq\langle n\rangle$ and functions $h: \mathcal{M}_{A} \times \mathcal{M}_{B} \longrightarrow \mathcal{M}_{A \cup B}$ for $A, B \subseteq\langle n\rangle$, such that if ${ }^{a} b$ denotes $h(a, b) \cdot b$ for $a \in \mathcal{M}_{A}$ and $b \in \mathcal{M}_{B}$ with $A \subseteq B$, then for all $a, a^{\prime} \in \mathcal{M}_{A}, b, b^{\prime} \in \mathcal{M}_{B}, c \in \mathcal{M}_{C}$ and $i, j \in\langle n\rangle$, the following conditions hold:

$$
\begin{aligned}
& \mu_{i}(a)=a \text { if } i \notin A, \\
& \mu_{i} \mu_{j}(a)=\mu_{j} \mu_{i}(a), \\
& \mu_{i} h(a, b)=h\left(\mu_{i}(a), \mu_{i}(b)\right), \\
& h(a, b)=h\left(\mu_{i}(a), b\right)=h\left(a, \mu_{i}(b)\right) \text { if } i \in A \cap B, \\
& h\left(a, a^{\prime}\right)=\left[a, a^{\prime}\right] \\
& h(a, b)=h(b, a)^{-1}, \\
& h(a, b)=1 \text { if } a=1 \text { or } b=1, \\
& h\left(a a^{\prime}, b\right)={ }^{a} h\left(a^{\prime}, b\right) h(a, b), \\
& h\left(a, b b^{\prime}\right)=h(a, b)^{b} h\left(a, b^{\prime}\right), \\
& { }^{a} h\left(h\left(a^{-1}, b\right), c\right)^{c} h\left(h\left(c^{-1}, a\right), b\right)^{b} h\left(h\left(b^{-1}, c\right), a\right)=1, \\
& { }^{a} h(b, c)=h\left({ }^{a} b,{ }^{a} c\right) \text { if } A \subseteq B \cap C .
\end{aligned}
$$

A morphism of crossed $n$-cubes, $\left\{\mathcal{M}_{A}\right\} \rightarrow\left\{\mathcal{M}_{A}^{\prime}\right\}$, is a family of group homomorphisms $\left\{f_{A}: \mathcal{M}_{A} \rightarrow \mathcal{M}_{A}^{\prime}, A \subseteq\langle n\rangle\right\}$ commuting with the $\mu_{i}$ and the $h$-functions. This gives a category of crossed $n$-cubes of groups which will be denoted by $\mathrm{Crs}^{n}$. 
Examples.

(i) A crossed 1-cube is the same as a crossed module (see Section 1).

(ii) A crossed 2-cube is the same as a crossed square (for the definition see [4]). The detailed reformulation is easy.

(iii) Let $G$ be a group and $N_{1}, \ldots, N_{n}$ be normal subgroups of $G$. Let $\mathcal{M}_{A}=$ $\bigcap_{i \in A} N_{i}$ for $A \subseteq\langle n\rangle$ (we also note that then $\mathcal{M}_{\emptyset}=G$ ); if $i \in\langle n\rangle$, define $\mu_{i}: \mathcal{M}_{A} \stackrel{i \in A}{\longrightarrow} \mathcal{M}_{A \backslash\{i\}}$ to be the inclusion and given $A, B \subseteq\langle n\rangle$, let $h: \mathcal{M}_{A} \times$ $\mathcal{M}_{B} \rightarrow \mathcal{M}_{A \cup B}$ be given by the commutator: $h(a, b)=[a, b]$ for $a \in \mathcal{M}_{A}, b \in$ $\mathcal{M}_{B}$ (here, of course, $\mathcal{M}_{A \cup B}=\mathcal{M}_{A} \cap \mathcal{M}_{B}$ ). Then $\left\{\mathcal{M}_{A}: A \subseteq\langle n\rangle, \mu_{i}, h\right\}$ is a crossed $n$-cube, called the inclusion crossed $n$-cube given by the normal $(n+1)$-ad of groups $\left(G ; N_{1}, \ldots, N_{n}\right)$.

Ellis and Steiner [11] prove that $C r s^{n}$ is equivalent to the category of cat $^{n}$-groups introduced by Loday who proved that equivalence for $n=1,2$, [17].

For a given crossed $n$-cube $\mathcal{M}$, there is an associated cat ${ }^{n}$-group and hence on applying the crossed module nerve structure $E$, examined in Section 1 , in the $n$ independent directions, this construction leads naturally to an $n$-simplicial group, called the multinerve of the crossed $n$-cube $\mathcal{M}$ and denoted by $\mathfrak{N e r}(\mathcal{M})$. Taking the diagonal of this $n$-simplicial group gives a simplicial group denoted by $E^{(n)}(\mathcal{M})_{*}$, (see $[25])$.

Proposition 11. Let $\mathcal{M}$ be an inclusion crossed $n$-cube given by a normal $(n+1)$-ad of groups $\left(F ; R_{1}, \ldots, R_{n}\right)$ and $k \geq 2$. Then there is a crossed $n$-cube $\mathcal{B}_{k}(\mathcal{M})$ given by:

(a) for $A \subseteq\langle n\rangle$

$$
\mathcal{B}_{k}(\mathcal{M})_{A}=\bigcap_{i \in A} R_{i} / D_{k}(F ; A)
$$

(b) if $j \in\langle n\rangle$, the homomorphism $\widetilde{\mu}_{j}: \mathcal{B}_{k}(\mathcal{M})_{A} \rightarrow \mathcal{B}_{k}(\mathcal{M})_{A \backslash\{j\}}$ is induced by the inclusion homomorphism $\mu_{j}$.

(c) representing an element in $\mathcal{B}_{k}(\mathcal{M})_{A}$ by $\bar{x}$ where $x \in \cap_{i \in A} R_{i}$ (the bar denotes a coset), and for $A, B \subseteq\langle n\rangle$, the map $\widetilde{h}: \mathcal{B}_{k}(\mathcal{M})_{A} \times \mathcal{B}_{k}(\mathcal{M})_{B} \rightarrow \mathcal{B}_{k}(\mathcal{M})_{A \cup B}$ is given by $\widetilde{h}(\bar{x}, \bar{y})=\overline{h(x, y)}=\overline{[x, y]}$ for all $\bar{x} \in \mathcal{B}_{k}(\mathcal{M})_{A}, \bar{y} \in \mathcal{B}_{k}(\mathcal{M})_{B}$.

Proof. In our notation

$$
D_{k}(F ; A)=\prod_{A_{1} \cup A_{2} \cup \cdots \cup A_{k}=A}\left[\bigcap_{i \in A_{1}} R_{i},\left[\bigcap_{i \in A_{2}} R_{i}, \ldots,\left[\underset{i \in A_{k-1}}{\cap} R_{i}, \underset{i \in A_{k}}{\cap} R_{i}\right] \ldots\right]\right], \quad A \subseteq\langle n\rangle .
$$

Since

$$
\begin{aligned}
{\left[\cap_{i \in A_{1}} R_{i},\left[\cap_{i \in A_{2}} R_{i}, \ldots,\right.\right.} & {\left.\left.\left[\bigcap_{i \in A_{k-1}} R_{i}, \underset{i \in A_{k}}{\cap} R_{i}\right] \ldots\right]\right] \subseteq } \\
& {\left[\underset{i \in A_{1} \backslash\{j\}}{\cap} R_{i},\left[\underset{i \in A_{2} \backslash\{j\}}{\cap} R_{i}, \ldots,\left[\bigcap_{i \in A_{k-1} \backslash\{j\}}^{\cap} R_{i}, \underset{i \in A_{k} \backslash\{j\}}{\cap} R_{i}\right] \ldots\right]\right] }
\end{aligned}
$$


for $A_{1} \cup \cdots \cup A_{k}=A \subseteq\langle n\rangle$, the inclusion $\mu_{j}: \bigcap_{i \in A} R_{i} \hookrightarrow \underset{i \in A \backslash\{j\}}{\bigcap} R_{i}$ induces the homomorphism $\widetilde{\mu_{j}}: \mathcal{B}_{k}(\mathcal{M})_{A} \rightarrow \mathcal{B}_{k}(\mathcal{M})_{A \backslash\{j\}}$ for all $j \in\langle n\rangle$.

Now we are only left to show that the function $\widetilde{h}: \mathcal{B}_{k}(\mathcal{M})_{A} \times \mathcal{B}_{k}(\mathcal{M})_{B} \rightarrow$ $\mathcal{B}_{k}(\mathcal{M})_{A \cup B}$ for $A, B \subseteq\langle n\rangle$ is well defined. In fact, let $x^{\prime} \in \cap_{i \in A} R_{i}, y^{\prime} \in \underset{i \in B}{\cap} R_{i}$ be such that

$$
x x^{\prime-1} \in \prod_{A_{1} \cup \cdots \cup A_{k}=A}\left[\underset{i \in A_{1}}{\cap} R_{i},\left[\underset{i \in A_{2}}{\cap} R_{i}, \ldots,\left[\underset{i \in A_{k-1}}{\cap} R_{i}, \underset{i \in A_{k}}{\cap} R_{i}\right] \ldots\right]\right]
$$

and

$$
y y^{\prime-1} \in \prod_{A_{1} \cup \cdots \cup A_{k}=B}\left[\bigcap_{i \in A_{1}} R_{i},\left[\underset{i \in A_{2}}{\cap} R_{i}, \ldots,\left[\underset{i \in A_{k-1}}{\cap} R_{i}, \bigcap_{i \in A_{k}} R_{i}\right] \ldots\right]\right] .
$$

The inclusion

$$
\left[\cap_{i \in A} R_{i}, \bigcap_{i \in B} R_{i}\right] \subseteq \bigcap_{i \in A \cup B} R_{i}
$$

for all $A, B \subseteq\langle n\rangle$ implies that

$$
\begin{aligned}
{[x, y]\left[x^{\prime}, y^{\prime}\right]^{-1} } & =x y x^{-1} y^{-1} y^{\prime} x^{\prime} y^{\prime-1} x^{\prime-1} \\
& =x y^{\prime}\left[y^{\prime-1} y, x^{-1}\right] y^{\prime-1} x^{-1} x\left[y^{\prime}, x^{-1} x^{\prime}\right] x^{-1} \\
& \in \prod_{A_{1} \cup \cdots \cup A_{k}=A \cup B}\left[\bigcap_{i \in A_{1}} R_{i},\left[\bigcap_{i \in A_{2}} R_{i}, \ldots,\left[\underset{i \in A_{k-1}}{\cap} R_{i}, \underset{i \in A_{k}}{\cap} R_{i}\right] \ldots\right]\right]
\end{aligned}
$$

so $\overline{h(x, y)}=\overline{h\left(x^{\prime}, y^{\prime}\right)}$ and $\widetilde{h}$ is well defined. The verification that $\mathcal{B}_{k}(\mathcal{M})$ is a crossed $n$-cube is routine and is left as an exercise.

Remark. The functor $\mathcal{B}_{2}$ coincides on the subcategory of inclusion crossed $n$-cubes with the abelianization functor $A b$ from the category $\operatorname{Crs}^{n}$ to the category $\mathfrak{A} b C r s^{n}$ of abelian crossed $n$-cubes of groups (i.e. crossed $n$-cubes all of whose $h$ maps are trivial), considered for $n=1$ in Section 1 and defined in general by the following way: for any $\mathcal{M}=\left\{\mathcal{M}_{A}: A \subseteq\langle n\rangle, \mu_{i}, h\right\}$ in $C r s^{n}, A b(\mathcal{M})$ is an abelian crossed $n$-cube given by:

(a) for $A \subseteq\langle n\rangle$

$$
A b(\mathcal{M})_{A}=\frac{\mathcal{M}_{A}}{\prod_{\substack{B, C \subseteq\langle n\rangle \\ B \cup C=A}} D_{B, C}}
$$

where $D_{B, C}$ is the subgroup of $\mathcal{M}_{A}$ generated by the elements $h(b, c)$, $h: \mathcal{M}_{B} \times \mathcal{M}_{C} \rightarrow \mathcal{M}_{B \cup C=A}$ for all $b \in \mathcal{M}_{B}, c \in \mathcal{M}_{C}$.

(b) if $i \in\langle n\rangle$, the homomorphism $\widetilde{\mu}_{i}: A b(\mathcal{M})_{A} \rightarrow A b(\mathcal{M})_{A \backslash\{i\}}$ is induced by the homomorphism $\mu_{i}$.

(c) for $A, B \subseteq\langle n\rangle$, the function $\widetilde{h}: A b(\mathcal{M})_{A} \times A b(\mathcal{M})_{B} \rightarrow A b(\mathcal{M})_{A \cup B}$ is induced by $h$ and therefore is trivial.

The functor $A b: C r s^{n} \rightarrow \mathfrak{A} b C r s^{n}$ is left adjoint to the inclusion functor $i: \mathfrak{A} b C r s^{n} \hookrightarrow C r s^{n}$ as is easily checked. 
For any inclusion crossed $n$-cube $\mathcal{M}$ given by a normal $(n+1)$-ad of groups $\left(F ; R_{1}, \ldots, R_{n}\right)$ and $k \geq 2$, there is a natural morphism of crossed $n$-cubes $\mathcal{M} \rightarrow$ $\mathcal{B}_{k}(\mathcal{M})$ inducing the natural fibration of simplicial groups $E^{(n)}(\mathcal{M})_{*} \stackrel{\Delta_{*}^{n, k}}{\rightarrow} E^{(n)}\left(\mathcal{B}_{k}(\mathcal{M})\right)_{*}$ defined by

$$
\Delta_{m}^{n, k}\left(x_{1}, \ldots, x_{l}\right)=\left(\overline{x_{1}}, \ldots, \overline{x_{l}}\right)
$$

for all $\left(x_{1}, \ldots, x_{l}\right) \in E^{(n)}(\mathcal{M})_{m}=\left(\bigcap_{i \in A_{1}} R_{i}\right) \rtimes \cdots \rtimes\left(\cap_{i \in A_{l}}^{\cap} R_{i}\right)$ and $m \geq 0$, where $A_{1}, \ldots, A_{l} \subseteq\langle n\rangle$ and $l=(m+1)^{n}$. It is easy to see that $\operatorname{Ker} \Delta_{m}^{n, k}=D_{k}\left(F ; A_{1}\right) \rtimes$ $\cdots \rtimes D_{k}\left(F ; A_{l}\right)$.

Let us consider a simplicial group $G_{*}$ and, applying the functor $Z_{k}$ dimension-wise, denote the resulting simplicial group by $Z_{k} G_{*}$.

Proposition 12. Let $\mathcal{M}$ be an inclusion crossed $n$-cube given by a normal $(n+1)$-ad of groups $\left(F ; R_{1}, \ldots, R_{n}\right)$ and $k \geq 2$. Then there is an isomorphism of simplicial groups

$$
Z_{k} E^{(n)}(\mathcal{M})_{*} \cong E^{(n)}\left(\mathcal{B}_{k}(\mathcal{M})\right)_{*}
$$

Proof. For any inclusion crossed module $R \hookrightarrow F$, It is easy to check the following equalities in the group $R \rtimes \cdots \rtimes R \rtimes F$ :

$$
\begin{aligned}
& {\left[(1, \ldots, 1, x),\left(1, \ldots, 1, x^{\prime}\right)\right]=\left(1, \ldots, 1,\left[x, x^{\prime}\right]\right)} \\
& {[(1, \ldots, 1, \underset{s}{r}, 1, \ldots, 1),(1, \ldots, 1, x)]=(1, \ldots, 1,[r, x], 1, \ldots, 1)} \\
& {\left[(1, \ldots, 1, \underset{s}{r}, 1, \ldots, 1),\left(1, \ldots, 1, r_{t}^{\prime}, 1, \ldots, 1\right)\right]=\left(1, \ldots, 1, \underset{\min \{s, t\}}{\left[r, r^{\prime}\right]}, 1, \ldots, 1\right)}
\end{aligned}
$$

for all $x, x^{\prime} \in F, r, r^{\prime} \in R$.

There are further generalisation of these equalities, namely for any inclusion crossed $n$-cube $\mathcal{M}$ given by the normal $n+1$-ad of groups $\left(F, R_{1}, \ldots, R_{n}\right)$ one has the following facts, the proof of which is routine and will be omitted.

(A) Let $s$ and $t$ be any fixed elements of the set $\left\langle(m+1)^{n}\right\rangle$. Then there exists a unique $\lambda=\lambda(s, t) \in\left\langle(m+1)^{n}\right\rangle$ such that $A_{\lambda}=A_{s} \cup A_{t}$ and in the group $E^{(n)}(\mathcal{M})_{m}$ there holds the equality:

$$
[(1, \ldots, 1, \underset{s}{x}, 1, \ldots, 1),(1, \ldots, 1, \underset{t}{y}, 1, \ldots, 1)]=(1, \ldots, \underset{\lambda}{1,} \underset{\lambda}{x}, y], 1, \ldots, 1)
$$

for all $x \in \bigcap_{i \in A_{s}} R_{i}, y \in \bigcap_{i \in A_{t}} R_{i}$.

(B) Let $s \in\left\langle(m+1)^{n}\right\rangle$ and $A, B \subseteq A_{s}$ with $A \cup B=A_{s}$. Then there exists $p, q \in\left\langle(m+1)^{n}\right\rangle$ such that $A_{p}=A, A_{q}=B$ and $\lambda(p, q)=s$.

We only have to show the equality

$$
\Gamma_{k}\left(E^{(n)}(\mathcal{M})_{m}\right)=\operatorname{Ker} \Delta_{m}^{n, k}
$$

which will be done by induction on $k$, using facts $(\mathrm{A})$ and $(\mathrm{B})$ above.

Let $k=1$, then it is clear that $\Gamma_{1}\left(E^{(n)}(\mathcal{M})_{m}\right)=\operatorname{Ker} \Delta_{m}^{n, 1}$.

Proceeding by induction, we suppose that (1) is true for $k-1$ and we will prove it for $k$. 
First we will show the inclusion $\operatorname{Ker} \Delta_{m}^{n, k} \subseteq \Gamma_{k}\left(E^{(n)}(\mathcal{M})_{m}\right)$. It is sufficient to show $1 \rtimes \cdots \rtimes 1 \rtimes D_{k}\left(F, A_{s}\right) \rtimes 1 \rtimes \cdots \rtimes 1 \subseteq \Gamma_{k}\left(E^{(n)}(\mathcal{M})_{m}\right) \quad$ for all $\quad s \in\left\langle(m+1)^{n}\right\rangle$. In fact, any generator $w$ of $D_{k}\left(F, A_{s}\right)$ has the form $w=[x, y]$, where $x \in \underset{i \in A}{\cap} R_{i}$, $y \in D_{k-1}(F, B)$ and $A \cup B=A_{s}$.

Now (B) implies that there exist $p, q \in\left\langle(m+1)^{n}\right\rangle$ such that $A_{p}=A, A_{q}=B$ and $\lambda(p, q)=s$. Thus we have

$$
[(1, \ldots, 1, \underset{p}{x}, 1, \ldots, 1),(1, \ldots, 1, \underset{q}{y}, 1, \ldots, 1)]=(1, \ldots, 1, \underset{s}{w}, 1, \ldots, 1),
$$

which means that

$$
1 \rtimes \cdots \rtimes 1 \rtimes D_{k}\left(F, A_{s}\right) \rtimes 1 \rtimes \cdots \rtimes 1 \subseteq\left[E^{(n)}(\mathcal{M})_{m}, \operatorname{Ker} \Delta_{m}^{n, k-1}\right] .
$$

Therefore by the inductive hypothesis we obtain

$1 \rtimes \cdots \rtimes 1 \rtimes D_{k}\left(F, A_{s}\right) \rtimes 1 \rtimes \cdots \rtimes 1 \subseteq\left[E^{(n)}(\mathcal{M})_{m}, \Gamma_{k-1}\left(E^{(n)}(\mathcal{M})_{m}\right)\right]=\Gamma_{k}\left(E^{(n)}(\mathcal{M})_{m}\right)$.

Finally we will show the inverse inclusion $\Gamma_{k}\left(E^{(n)}(\mathcal{M})_{m}\right) \subseteq \operatorname{Ker} \Delta_{m}^{n, k}$. In fact, any generator $w$ of $\Gamma_{k}\left(E^{(n)}(\mathcal{M})_{m}\right)$ could be written as $w=\left[w_{1}, w_{2}\right]$, where $w_{1} \in$ $E^{(n)}(\mathcal{M})_{m}$ and $w_{2} \in \Gamma_{k-1}\left(E^{(n)}(\mathcal{M})_{m}\right)$. Using again the inductive hypothesis we have $w_{2} \in \operatorname{Ker} \Delta_{m}^{n, k-1}$. Thus

$$
\begin{aligned}
& w_{1}=\prod_{s=1}^{(m+1)^{n}}\left(1, \ldots, 1, x_{s}, 1, \ldots, 1\right), \quad x_{s} \in \bigcap_{i \in A_{s}} R_{i}, \\
& w_{2}=\prod_{t=1}^{(m+1)^{n}}\left(1, \ldots, 1, y_{t}, 1, \ldots, 1\right), \quad y_{t} \in D_{k-1}\left(F, A_{t}\right) .
\end{aligned}
$$

It is certain that $\left[x_{s}, y_{t}\right] \in D_{k}\left(F, A_{s} \cup A_{t}\right)$. Then (A) implies that we have

$$
\begin{gathered}
{\left[\left(1, \ldots, 1, x_{s}, 1, \ldots, 1\right),\left(1, \ldots, 1, y_{t}, 1, \ldots, 1\right)\right]=\left(1, \ldots, 1,\left[x_{s}, y_{t}\right], 1, \ldots, 1\right)} \\
\in 1 \rtimes \cdots \rtimes 1 \rtimes D_{k}\left(F, A_{\lambda(s, t)}\right) \rtimes 1 \rtimes \cdots \rtimes 1 \subseteq \operatorname{Ker} \Delta_{m}^{n, k} .
\end{gathered}
$$

Then the Witt-Hall identities on commutators implies that $w \in \operatorname{Ker} \Delta_{m}^{n, k}$.

\section{Non-ABElian MAPPING CONE COMPlex}

A complex of (non-abelian) groups $\left(A_{*}, d_{*}\right)$ of length $n$ is a sequence of group homomorphisms

$$
A_{n} \stackrel{d_{n}}{\longrightarrow} A_{n-1} \stackrel{d_{n-1}}{\longrightarrow} \cdots \stackrel{d_{1}}{\longrightarrow} A_{0}
$$

such that $\operatorname{Im} d_{i+1}$ is normal in Ker $d_{i}$. Now we recall the following definition from [17].

Let $f:\left(A_{*}, d_{*}\right) \rightarrow\left(B_{*}, d_{*}^{\prime}\right)$ be a morphism of chain complexes of groups. Let $f$ satisfy the following conditions $(*)$ :

each $f_{i}: A_{i} \rightarrow B_{i}$ is a crossed module and 
the maps $\left(d_{i}, d_{i}^{\prime}\right)$ form a morphism of crossed modules.

Then the mapping cone of $f$ is a complex of (non-abelian) groups $\left(C_{*}(f), \partial_{*}\right)$ defined by $C_{i}(f)=A_{i-1} \rtimes B_{i}$, where the action of $B_{i}$ on $A_{i-1}$ is induced by the action of $B_{i-1}$ on $A_{i-1}$ via the homomorphism $d_{i}^{\prime}$; and

$$
\partial_{i}(a, b)=\left(d_{i-1}(a)^{-1}, f_{i-1}(a) d_{i}^{\prime}(b)\right)
$$

for all $a \in A_{i-1}, b \in B_{i}$. Then by [17], Proposition 3.2, there is a long exact sequence of groups.

$$
\cdots \longrightarrow H_{i}\left(A_{*}\right) \longrightarrow H_{i}\left(B_{*}\right) \longrightarrow H_{i}\left(C_{*}(f)\right) \longrightarrow H_{i-1}\left(A_{*}\right) \longrightarrow \cdots .
$$

Now let us consider a morphism of pseudosimplicial groups $\alpha:\left(G_{*}, d_{i}^{*}, s_{i}^{*}\right) \rightarrow$ $\left(H_{*}, d_{i}^{\prime *}, s_{i}^{*}\right)$ satisfying conditions $(* *)$ :

each $\alpha_{n}: G_{n} \rightarrow H_{n}$ is a crossed module

and

the maps $\left(d_{i}^{*}, d_{i}^{\prime *}\right)$ and $\left(s_{i}^{*}, s_{i}^{\prime *}\right)$ form morphisms of crossed modules.

Define a new pseudosimplicial group $M_{*}(\alpha)$ in the following way:

$$
M_{n}(\alpha)=\underbrace{G_{n} \rtimes G_{n} \rtimes \cdots \rtimes G_{n}}_{n \text {-times }} \rtimes H_{n},
$$

$d_{0}^{n}\left(g_{1}, \ldots, g_{n}, h\right)=\left(d_{0}^{n}\left(g_{2}\right), \ldots, d_{0}^{n}\left(g_{n}\right), d_{0}^{\prime n}(h)\right)$,

$d_{i}^{n}\left(g_{1}, \ldots, g_{n}, h\right)=\left(d_{i}^{n}\left(g_{1}\right), \ldots, d_{i}^{n}\left(g_{i}\right) d_{i}^{n}\left(g_{i+1}\right), \ldots, d_{i}^{n}\left(g_{n}\right), d_{i}^{\prime n}(h)\right), \quad 0<i<n$,

$d_{n}^{n}\left(g_{1}, \ldots, g_{n}, h\right)=\left(d_{n}^{n}\left(g_{1}\right), \ldots, d_{n}^{n}\left(g_{n-1}\right), \alpha_{n-1} d_{n}^{n}\left(g_{n}\right) d_{n}^{\prime n}(h)\right)$,

$s_{i}^{n}\left(g_{1}, \ldots, g_{n}, h\right)=\left(s_{i}^{n}\left(g_{1}\right), \ldots, s_{i}^{n}\left(g_{i}\right), 1, s_{i}^{n}\left(g_{i+1}\right), \ldots, s_{i}^{n}\left(g_{n}\right), s_{i}^{\prime n}(h)\right), \quad 0 \leq i \leq n$.

It is easy to see that the induced morphism $\widetilde{\alpha}: N G_{*} \rightarrow N H_{*}$, where $N G_{*}$ and $N H_{*}$ are the Moore complexes of $G_{*}$ and $H_{*}$ respectively, satisfies the conditions $(*)$. Therefore one can consider the mapping cone complex $C_{*}(\widetilde{\alpha})$ of $\widetilde{\alpha}$.

Proposition 13. The natural morphism of complexes $\kappa: N M_{*}(\alpha) \rightarrow C_{*}(\widetilde{\alpha})$, given by $\kappa_{n}\left(g_{1}, g_{2}, \ldots, g_{n}, h\right)=\left(d_{n}^{n}\left(g_{n}\right), h\right), n \geq 0$ induces an isomorphism of groups

$$
\pi_{n}\left(M_{*}(\alpha)\right) \cong H_{n}\left(C_{*}(\widetilde{\alpha})\right), \quad n \geq 0 .
$$

Proof. The verification that $\kappa_{n}, n \geq 0$ is a homomorphism and commuting with differentials is easy. Let $(g, h) \in N G_{n-1} \rtimes N H_{n}=C_{n}(\widetilde{\alpha})$, then it is easy to check that $\left(s_{0}^{n-1}(g)^{\epsilon(n-1)}, \ldots, s_{n-2}^{n-1}(g)^{-1}, s_{n-1}^{n-1}(g), h\right) \in N M_{n}(\alpha)$, where $\epsilon(i)=(-1)^{i}$. It is clear that $\kappa_{n}\left(s_{0}^{n-1}(g)^{\epsilon(n-1)}, \ldots, s_{n-2}^{n-1}(g)^{-1}, s_{n-1}^{n-1}(g), h\right)=(g, h)$. Hence $\kappa_{n}$ is surjective for all $n \geq 0$.

Consider the kernel complex $\left(\mathfrak{G}_{*}, \bar{\partial}_{*}\right)$ of $\kappa$. Note that $\operatorname{Im} \bar{\partial}_{n}$ is not normal in Ker $\bar{\partial}_{n-1}$ in general, $\mathfrak{G}_{0}=1$ and

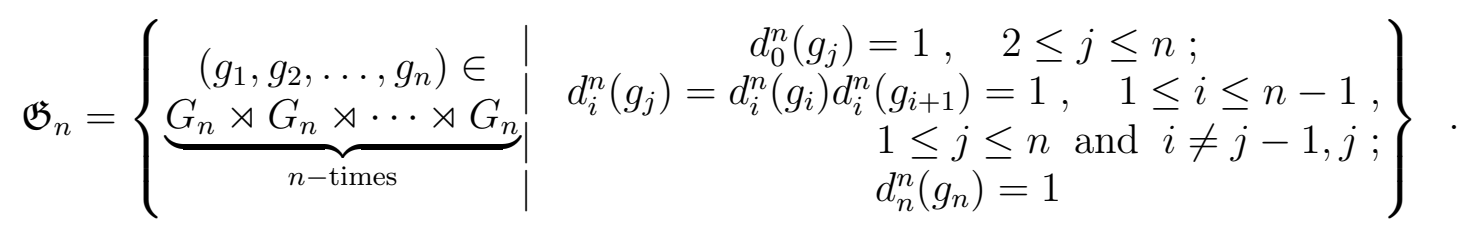


Furthermore, it is easy to check that for an element $\left(g_{1}, \ldots, g_{n-1}\right) \in \operatorname{Ker} \overline{\partial_{n-1}}$, the element $\left(g_{1}^{\prime}, \ldots, g_{n-1}^{\prime}, g_{n}^{\prime}\right)$, defined by the formulae:

$$
g_{i}^{\prime}=\left\{\begin{array}{l}
s_{n-1}^{n-1}\left(g_{i}\right) s_{n-2}^{n-1}\left(g_{i}^{-1}\right) \cdots s_{i}^{n-1}\left(g_{i}^{\epsilon(n-i-1)}\right) s_{i-1}^{n-1}\left(g_{i}^{\epsilon(n-i)} g_{i+1}^{\epsilon(n-i)} \cdots g_{n-1}^{\epsilon(n-i)}\right), i \text { even } \\
s_{i-1}^{n-1}\left(g_{n-1}^{\epsilon(n-i)} \cdots g_{i+1}^{\epsilon(n-i)} g_{i}^{\epsilon(n-i)}\right) s_{i}^{n-1}\left(g_{i}^{\epsilon(n-i-1)}\right) \cdots s_{n-2}^{n-1}\left(g_{i}^{-1}\right) s_{n-1}^{n-1}\left(g_{i}\right), i \text { odd }
\end{array}\right.
$$

for all $1 \leq i \leq n-1$ and $g_{n}^{\prime}=1$, belongs to $\mathfrak{G}_{n}$ and

$$
\overline{\partial_{n}}\left(g_{1}^{\prime}, \ldots, g_{n-1}^{\prime}, g_{n}^{\prime}\right)=\left(g_{1}, \ldots, g_{n-1}\right) \text {. }
$$

Now the proposition follows from the long exact homology sequence induced by the short exact sequence of complexes $1 \longrightarrow \mathfrak{G}_{*} \longrightarrow N M_{*}(\alpha) \stackrel{\kappa}{\longrightarrow} C_{*}(\widetilde{\alpha}) \longrightarrow 1$.

Given a pseudosimplicial group $G_{*}$, we will say that the length of $G_{*}$ is $\leq n$, denoted by $l\left(G_{*}\right) \leq n$ if $N G_{i}=1$ for $i>n$.

Remark. Let $\alpha:\left(G_{*}, d_{i}^{*}, s_{i}^{*}\right) \rightarrow\left(H_{*}, d_{i}^{*}, s_{i}^{*}\right)$ be a morphism of pseudosimplicial groups satisfying the conditions $(* *)$ and $n \geq 2$. Suppose $l\left(G_{*}\right) \leq n-1$ and $l\left(H_{*}\right) \leq n-1$. Consider an element $\left(g_{1}, g_{2}, \ldots, g_{k}, h\right) \in N M_{k}(\alpha), k>n$, then

$$
\begin{aligned}
& d_{0}^{k}\left(g_{j}\right)=1, \quad 2 \leq j \leq k, \\
& d_{i}^{k}\left(g_{j}\right)=d_{i}^{k}\left(g_{i}\right) d_{i}^{k}\left(g_{i+1}\right)=1, \quad 1 \leq i \leq k-1, \quad 1 \leq j \leq k \text { and } i \neq j-1, j, \\
& d_{i}^{\prime k}(h)=1, \quad 0 \leq i \leq k-1 .
\end{aligned}
$$

By Lemma 8 one can easily show that $g_{i}=1,1 \leq i \leq k$ and $h=1$, meaning $N M_{k}(\alpha)=1$ for $k>n$. Thus $l\left(M_{*}(\alpha)\right) \leq n$.

Now using the mapping cone construction, for a given crossed $n$-cube $\mathcal{M}$, we construct inductively a complex of groups $C_{*}(\mathcal{M})$ of length $n$, always having in mind that $\mathcal{M}$ is thought as a crossed module of crossed $(n-1)$-cubes, $\mathcal{M}_{1} \rightarrow \mathcal{M}_{0}$ (Proposition 5, [25]). In fact, for $n=1$, and $\mathcal{M}=(M \stackrel{\mu}{\rightarrow} P), C_{*}(\mathcal{M})$ is the complex $M \rightarrow P$ of length 1 . Let $n=2$ and $\mathcal{M}$ be a crossed square, considered as a crossed module of crossed modules or a morphism of complexes of length 1 satisfying the conditions $(*)$. The construction above gives a complex $C_{*}(\mathcal{M})$ of length 2. (This has a 2-crossed module structure, [6], as noted by Conduché, see also [21].) Proceeding by induction, suppose for any crossed $(n-1)$-cube $\mathcal{M}$ we have constructed a complex $C_{*}(\mathcal{M})$ of length $n-1$. Now let $\mathcal{M}$ be a crossed $n$-cube and consider it as a crossed module of crossed $(n-1)$-cubes $\mathcal{M}_{1} \rightarrow \mathcal{M}_{0}$, which implies a morphism of complexes of groups $C_{*}\left(\mathcal{M}_{1}\right) \stackrel{\delta}{\longrightarrow} C_{*}\left(\mathcal{M}_{0}\right)$ of length $n-1$ satisfying the conditions $(*)$. So using again the above-mentioned construction we obtain a complex of groups $C_{*}(\mathcal{M})=C_{*}(\delta)$ of length $n$.

Proposition 14. [17] Let $\mathcal{M}$ be a crossed $n$-cube of groups. Then $l\left(E^{n}(\mathcal{M})_{*}\right) \leq n$ and there is a natural morphism of complexes $N E^{(n)}(\mathcal{M})_{*} \rightarrow C_{*}(\mathcal{M})$ which induces isomorphisms of groups

$$
\begin{aligned}
& \pi_{i}\left(E^{(n)}(\mathcal{M})_{*}\right) \cong H_{i}\left(C_{*}(\mathcal{M})\right), \quad i \geq 0 . \\
& \text { Moreover } \pi_{n}\left(E^{(n)}(\mathcal{M})_{*}\right) \cong \bigcap_{i=1}^{n} \operatorname{Ker}\left(\mathcal{M}_{\langle n\rangle} \stackrel{\mu_{i}}{\longrightarrow} \mathcal{M}_{\langle n\rangle \backslash\{i\}}\right) .
\end{aligned}
$$


Proof. This is obvious for $n=1$. Let $n=2$ and $\mathcal{M}$ be a crossed square respectively. If we consider $\mathcal{M}$ as a crossed module of crossed modules $\mathcal{M}_{1} \rightarrow \mathcal{M}_{0}$ inducing the natural morphism of simplicial groups $E^{(1)}\left(\mathcal{M}_{1}\right)_{*} \stackrel{\alpha}{\rightarrow} E^{(1)}\left(\mathcal{M}_{0}\right)_{*}$ satisfying the conditions $(* *)$, then by definition $E^{(2)}(\mathcal{M})_{*}=M_{*}(\alpha)$, and by Proposition 13 and the corresponding Remark, $l\left(E^{(2)}(\mathcal{M})_{*}\right) \leq 2$, and there exists a natural morphism of complexes $N E^{(2)}(\mathcal{M})_{*} \rightarrow C_{*}(\widetilde{\alpha})$ inducing an isomorphism

$$
\pi_{i}\left(E^{(2)}(\mathcal{M})_{*}\right) \cong H_{i}\left(C_{*}(\widetilde{\alpha})\right), \quad i \geq 0 .
$$

Clearly $C_{*}(\widetilde{\alpha}) \cong C_{*}(\mathcal{M})$.

Proceeding by induction, we suppose that the assertion is true for $n-1$ and we will show it for $n$.

Let us consider any crossed $n$-cube $\mathcal{M}$ as a crossed module of crossed $(n-1)$ cubes $\mathcal{M}_{1} \rightarrow \mathcal{M}_{0}$. This implies a morphism of simplicial groups $E^{(n-1)}\left(\mathcal{M}_{1}\right)_{*} \stackrel{\alpha}{\rightarrow}$ $E^{(n-1)}\left(\mathcal{M}_{0}\right)_{*}$ satisfying the conditions $(* *)$ and a morphism of complexes $C_{*}\left(\mathcal{M}_{1}\right) \stackrel{\delta}{\rightarrow}$ $C_{*}\left(\mathcal{M}_{0}\right)$ satisfying the conditions $(*)$. By definition $E^{(n)}(\mathcal{M})_{*}=M_{*}(\alpha)$, hence Proposition 13 and its Remark imply that $l\left(E^{(n)}(\mathcal{M})_{*}\right) \leq n$ and there exists a natural morphism of complexes $N E^{(n)}(\mathcal{M})_{*} \stackrel{\kappa}{\rightarrow} C_{*}(\widetilde{\alpha})$ inducing isomorphisms

$$
\pi_{i}\left(E^{(n)}(\mathcal{M})_{*}\right) \cong H_{i}\left(C_{*}(\widetilde{\alpha})\right), \quad i \geq 0 .
$$

Using the inductive hypothesis, there exist natural morphisms of complexes

$$
N E^{(n-1)}\left(\mathcal{M}_{1}\right)_{*} \stackrel{\kappa^{\prime}}{\rightarrow} C_{*}\left(\mathcal{M}_{1}\right) \text { and } N E^{(n-1)}\left(\mathcal{M}_{0}\right)_{*} \stackrel{\kappa^{\prime \prime}}{\rightarrow} C_{*}\left(\mathcal{M}_{0}\right),
$$

which induce isomorphisms

$$
\begin{aligned}
& \pi_{i}\left(E^{(n-1)}\left(\mathcal{M}_{1}\right)_{*}\right) \cong H_{i}\left(C_{*}\left(\mathcal{M}_{1}\right)\right), \\
& \pi_{i}\left(E^{(n-1)}\left(\mathcal{M}_{0}\right)_{*}\right) \cong H_{i}\left(C_{*}\left(\mathcal{M}_{0}\right)\right),
\end{aligned}
$$

for $i \geq 0$. It is easy to check that $\kappa^{\prime \prime} \widetilde{\alpha}=\delta \kappa^{\prime}$ and that $\left(\kappa_{i}^{\prime}, \kappa_{i}^{\prime \prime}\right)$ is a morphism of crossed modules for all $i \geq 0$. Then the natural morphism of complexes $C_{*}(\widetilde{\alpha}) \stackrel{\kappa^{\prime} \rtimes \kappa^{\prime \prime}}{\longrightarrow}$ $C_{*}(\delta)=C_{*}(\mathcal{M})$, by $(2)$ and the five lemma, induces $H_{i}\left(C_{*}(\widetilde{\alpha})\right) \cong H_{i}\left(C_{*}(\mathcal{M})\right), i \geq 0$. Therefore the morphism of complexes

$$
N E^{(n)}(\mathcal{M})_{*} \stackrel{\left(\kappa^{\prime} \rtimes \kappa^{\prime \prime}\right) \circ \kappa}{\longrightarrow} C_{*}(\mathcal{M})
$$

induces

$$
\pi_{i}\left(E^{(n)}(\mathcal{M})_{*}\right) \cong H_{i}\left(C_{*}(\mathcal{M})\right), \quad i \geq 0 .
$$

From these isomorphisms and the construction of $C_{*}(\mathcal{M})$ follows that

$$
\pi_{n}\left(E^{(n)}(\mathcal{M})_{*}\right) \cong \bigcap_{i=1}^{n} \operatorname{Ker}\left(\mathcal{M}_{\langle n\rangle} \stackrel{\mu_{i}}{\longrightarrow} \mathcal{M}_{\langle n\rangle \backslash\{i\}}\right) .
$$




\section{5. $n$-FOLD ČECH DERIVED FUNCTORS}

The Čech derived functors of group valued functors were introduced in [22] (see also [12] and, here, our Section 1) as an algebraic analogue of the Čech (co)homology construction of open covers of topological spaces with coefficients in sheaves of abelian groups (see [29]). It is well known that the Cech cohomlogy of topological spaces with coefficients in sheaves is closely related to sheaf cohomology of topological spaces, in particular this relation is expressed by spectral sequences [29]. Some applications of Čech derived functors to group (co)homology theory and $K$ theory are given in $[22,23,24]$. In this section we generalise the notion of the Cech derived functors to that of the $n$-fold Cech derived functors of an endofunctor on the category of groups. Based on this notion we get a new purely algebraic method for the investigation of higher integral homology of groups from a Hopf formula point of view and the further generalizations of these formulae.

Let us consider again the set $\langle n\rangle=\{1, \ldots, n\}$. The subsets of $\langle n\rangle$ are ordered by inclusion. This ordered set determines in the usual way a category $C_{n}$. For every pair $(A, B)$ of subsets with $A \subseteq B \subseteq\langle n\rangle$, there is the unique morphism $\rho_{B}^{A}: A \rightarrow B$ in $\underline{C_{n}}$. It is easy to see that any morphism in the category $C_{n}$, not an identity, is generated by $\rho_{B}^{A}$ for all $A \subseteq\langle n\rangle, A \neq\langle n\rangle, B=A \cup\{j\}, j \notin \overline{A \text {. }}$

An $n$-cube of groups is a functor $\mathfrak{F}: C_{n} \rightarrow \mathfrak{G} r, A \mapsto \mathfrak{F}_{A}, \rho_{B}^{A} \mapsto \alpha_{B}^{A}$. A morphism between $n$-cubes $\mathfrak{F}, \mathfrak{Q}: \underline{C_{n}} \rightarrow \mathfrak{G} r$ is a natural transformation $\kappa: \mathfrak{F} \rightarrow \mathfrak{Q}$.

WARNING: A crossed $n$-cube of groups gives an $n$-cube on forgetting structure, but note that there is a reversal of the role of the index $A$. The top corner of a crossed $n$-cube is $G_{\langle n\rangle}$, that in an $n$-cube is $\mathfrak{F}_{\emptyset}$. This is due to the fact that an $n$-cube of groups yields a crossed $n$-cube as a sort of generalized kernel.

Let $A \subseteq\langle n\rangle$ and consider two full subcategories of the category $\underline{C_{n}}$ : $\underline{C_{n}^{A}}$ is the category of all subsets of $\langle n\rangle$ containing the subset $A$ and $\underline{C_{n}^{\bar{A}}}$ is the category of all subsets of $\langle n\rangle$ not containing the subset $A$. For a given $n$-cube of groups $\mathfrak{F}$, and $A$ as above, denote by $\mathfrak{F}^{A}$ and $\mathfrak{F}^{\bar{A}}$ the functors induced by the restriction of the functor $\mathfrak{F}$ to the subcategories $C_{n}^{A}$ and $C_{n}^{\bar{A}}$ respectively. For a given morphism of $n$-cubes of groups $\kappa: \mathfrak{F} \rightarrow \mathfrak{Q}$ denote by $\kappa^{\bar{A}:} \mathfrak{F}^{A} \rightarrow \mathfrak{Q}^{A}$ the natural transformation induced by restriction of the natural transformation $\kappa$.

\section{Examples}

(a) Let $\left(F ; R_{1}, \ldots, R_{n}\right)$ be a normal $(n+1)$-ad of groups. These data naturally determines an $n$-cube of groups $\mathfrak{F}$ as follows: for any $A \subseteq\langle n\rangle$, let $\mathfrak{F}_{A}=F / \prod_{i \in A} R_{i}$; for an inclusion $A \subseteq B$, let $\alpha_{B}^{A}: \mathfrak{F}_{A} \rightarrow \mathfrak{F}_{B}$ be the natural homomorphism induced by $1_{F}$. This $n$-cube of groups will be called the $n$-cube of groups induced by the normal $(n+1)$-ad of groups, $\left(F ; R_{1}, \ldots, R_{n}\right)$. 
(b) Let $\left(G_{*}, d_{0}^{0}, G\right)$ be an augmented pseudosimplicial group. A natural $n$-cube of groups $\mathcal{G}(n): \underline{C_{n}} \rightarrow \mathfrak{G} r, n \geq 1$ is defined by the following way:

$$
\begin{gathered}
\mathcal{G}(n)_{A}=G_{n-1-|A|} \text { for all } A \subseteq\langle n\rangle, \\
\alpha_{A \cup\{j\}}^{A}=d_{k-1}^{n-1-|A|} \text { for all } A \neq\langle n\rangle, j \notin A,
\end{gathered}
$$

where $G_{-1}=G, \delta(k)=j$ and $\delta:\langle n-|A|\rangle \rightarrow\langle n\rangle \backslash A$ is the unique monotone bijection.

Given an $n$-cube of groups $\mathfrak{F}$. It is easy to see that there exists a natural homomorphism $\mathfrak{F}_{A} \stackrel{\alpha_{A}}{\longrightarrow} \lim _{B \supset A} \mathfrak{F}_{B}$ for any $A \subseteq\langle n\rangle, A \neq\langle n\rangle$.

Let $G$ be a group. An $n$-cube of groups $\mathfrak{F}$ will be called an $n$-presentation of the group $G$ if $\mathfrak{F}_{\langle n\rangle}=G$. An $n$-presentation $\mathfrak{F}$ of $G$ is called free if the group $\mathfrak{F}_{A}$ is free for all $A \neq\langle n\rangle$ and called exact if the homomorphism $\alpha_{A}$ is surjective for all $A \neq\langle n\rangle$. Note that a fibrant $n$-presentation of a group $G$ in the sense of Brown-Ellis [3] is the same as a free exact $n$-presentation of $G$ in our sense, for a construction of such, see [3].

Proposition 15. Let $\left(G_{*}, d_{0}^{0}, G\right)$ be an augmented pseudosimplicial group and suppose that $d_{0}^{0}$ induces a natural isomorphism $\pi_{0}\left(G_{*}\right) \stackrel{\widetilde{d_{0}^{0}}}{\rightarrow} G$.

(i) Then the $n$-cube of groups $\mathcal{G}(n), n \geq 1$, is induced by the normal $(n+1)$-ad of groups $\left(G_{n-1}\right.$, Ker $\left.d_{0}^{n-1}, \ldots, \operatorname{Ker} d_{n-1}^{n-1}\right)$ i.e.

$$
\mathcal{G}(n)_{A} \cong G_{n-1} / \prod_{i \in A} \operatorname{Ker} d_{i-1}^{n-1}, \quad A \subseteq\langle n\rangle .
$$

(ii) $\left(G_{*}, d_{0}^{0}, G\right)$ is aspherical if and only if the $n$-cube of groups $\mathcal{G}(n)$ is an exact $n$-presentation of the group $G$ for all $n \geq 1$.

Proof. (i) Straightforward from the following well-known fact on pseudosimplicial groups:

$$
d_{j}^{n}\left(\operatorname{Ker} d_{i}^{n}\right)=\operatorname{Ker} d_{i}^{n-1} \text { for } n>0,0 \leq i<j \leq n .
$$

(ii) It is well-known that asphericity of the augmented pseudosimplicial group $\left(G_{*}, d_{0}^{0}, G\right)$ is equivalent to the simplicial exactness of $\left(G_{*}, d_{0}^{0}, G\right)$, which certainly is equivalent to the fact that $\mathcal{G}(n)$ is an exact $n$-presentation of $G$ for all $n \geq 1$.

Remark. From Proposition 15(i) follows that if $\left(F_{*}, d_{0}^{0}, G\right)$ is an augmented pseudosimplicial group such that $d_{0}^{0}$ induces a natural isomorphism

$$
\pi_{0}\left(F_{*}\right) \stackrel{\widetilde{d_{0}^{0}}}{\rightarrow} G
$$

and $F_{i}, i \geq 0$ are free groups, then the normal $(n+1)$-ad of groups

$$
\left(F_{n-1}, \operatorname{Ker} d_{0}^{n-1}, \ldots, \operatorname{Ker} d_{n-1}^{n-1}\right)
$$

satisfies the conditions of Theorem BE (see also [3], [10]). Thus, if these conditions were sufficient, simplicial exactness (or asphericity) of $\left(F_{*}, d_{0}^{0}, G\right)$ would not be needed for getting the generalised Hopf formulas for higher homology of groups. It was this, in fact, that made us suspect that these BE conditions are not sufficient. 
Given an $n$-cube of groups $\mathfrak{F}$, a normal $(n+1)$-ad of groups $\left(F ; R_{1}, \ldots, R_{n}\right)$, where $F=\mathfrak{F}_{\emptyset}$ and $R_{i}=\operatorname{Ker} \alpha_{\{i\}}^{\emptyset}, i \in\langle n\rangle$ is called the normal $(n+1)$-ad of groups induced by $\mathfrak{F}$. If $\mathfrak{F}$ is an exact $n$-presentation of the group $\mathfrak{F}_{\langle n\rangle}$, then the normal $(n+1)$-ad of groups $\left(F ; R_{1}, \ldots, R_{n}\right)$ satisfies the following condition:

$$
F / \prod_{i \in A} R_{i} \cong \mathfrak{F}_{A} \text { for all } A \subseteq\langle n\rangle
$$

i.e. the $n$-cube of groups $\mathfrak{F}$ is induced by the normal $(n+1)$-ad of groups $\left(F ; R_{1}, \ldots, R_{n}\right)$ (see [15]).

Now let $\mathfrak{F}$ be an $n$-presentation of the group $G$. Applying $\check{C}$ (see Section 1 ) in the $n$-independent directions, this construction leads naturally to an augmented $n$ simplicial group. Taking the diagonal of this augmented $n$-simplicial group gives the augmented simplicial group $\left(\check{C}^{(n)}(\mathfrak{F})_{*}, \alpha, G\right)$ called an augmented $n$-fold $\check{C}$ ech complex for $\mathfrak{F}$, where $\alpha=\alpha_{\langle n\rangle}^{\emptyset}: \mathfrak{F}_{\emptyset} \rightarrow G$. In case $\mathfrak{F}$ is a free exact $n$-presentation of the group $G$, then $\left(\check{C}^{(n)}(\mathfrak{F})_{*}, \alpha, G\right)$ will be called an $n$-fold $\check{C}$ ech resolution of $G$.

Let $G, H$ be groups. Let $\mathfrak{F}$ and $\mathfrak{Q}$ be $n$-presentations of $G$ and $H$ respectively and $\lambda: G \rightarrow H$ a morphism of groups. A morphism $\kappa: \mathfrak{F} \rightarrow \mathfrak{Q}$ of $n$-cubes will be called an extension of the group morphism $\lambda$ if $\kappa_{\langle n\rangle}=\lambda$.

Theorem 16. Let $\mathfrak{F}$ and $\mathfrak{Q}$ be free and exact $n$-presentations of given groups $G$ and $H$ respectively. Then any morphism of groups $\lambda: G \rightarrow H$ can be extended to a morphism $\kappa: \mathfrak{F} \rightarrow \mathfrak{Q}$ of $n$-cubes of groups which naturally induces a morphism $\widetilde{\kappa}$ of simplicial groups

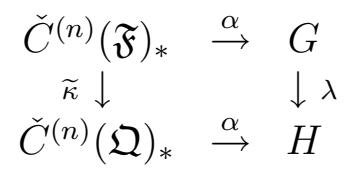

over $\lambda$. Furthermore, any two such extensions $\kappa, \pi: \mathfrak{F} \rightarrow \mathfrak{Q}$ of $\lambda$ induce simplicially homotopic morphisms $\widetilde{\kappa}, \widetilde{\pi}$ of simplicial groups, denoted by $\widetilde{\kappa} \simeq \widetilde{\pi}$.

Proof. We begin by showing the existence of a morphism of $n$-cubes of groups $\kappa$ : $\mathfrak{F} \rightarrow \mathfrak{Q}$ extending the morphism of groups $\lambda: G \rightarrow H$.

Since $\mathfrak{F}$ is free and $\mathfrak{Q}$ is exact, there exists a homomorphism $\kappa_{\langle n\rangle \backslash\{i\}}: \mathfrak{F}_{\langle n\rangle \backslash\{i\}} \rightarrow$ $\mathfrak{Q}_{\langle n\rangle \backslash\{i\}}$ for all $i \in\langle n\rangle$, such that $\alpha_{\langle n\rangle}^{\langle n\rangle \backslash i\}} \kappa_{\langle n\rangle \backslash\{i\}}=\lambda \alpha_{\langle n\rangle}^{\langle n\rangle\{i\}}$. Suppose for some $A \subseteq\langle n\rangle$ and for all $B \supset A, B \subseteq\langle n\rangle$, there exists a homomorphism $\kappa_{B}: \mathfrak{F}_{B} \rightarrow \mathfrak{Q}_{B}$ such that $\alpha_{C}^{B} \kappa_{B}=\kappa_{C} \alpha_{C}^{B}, C \supseteq B$. Then as an immediate consequence one has the induced homomorphism $\bar{\kappa}: \lim _{B \supset A} \mathfrak{F}_{B} \rightarrow \lim _{B \supset A} \mathfrak{Q}_{B}$. Using again the facts that $\mathfrak{F}$ is free and $\mathfrak{Q}$ is exact there exists a homomorphism $\kappa_{A}: \mathfrak{F}_{A} \rightarrow \mathfrak{Q}_{A}$ such that $\alpha_{A} \kappa_{A}=\bar{\kappa} \alpha_{A}$. Clearly the constructed morphism of $n$-cubes of groups $\kappa: \mathfrak{F} \rightarrow \mathfrak{Q}$ naturally induces a unique morphism of augmented $n$-simplicial groups and applying the diagonal gives a morphism of simplicial groups $\widetilde{\kappa}: \check{C}^{(n)}(\mathfrak{F})_{*} \rightarrow \check{C}^{(n)}(\mathfrak{Q})_{*}$ over the morphism $\lambda$.

We need to prove the remaining part of the assertion first in a particular case.

Particular Case. Let $\kappa, \pi: \mathfrak{F} \rightarrow \mathfrak{Q}$ be two extensions of the group morphism $\lambda: G \rightarrow H$ and $l \in\langle n\rangle$. Let $\kappa^{\{l\}}=\pi^{\{l\}}: \mathfrak{F}^{\{l\}} \rightarrow \mathfrak{Q}^{\{l\}}$, then the respective induced 
morphisms of simplicial groups $\widetilde{\kappa}, \widetilde{\pi}: \check{C}^{(n)}(\mathfrak{F})_{*} \rightarrow \check{C}^{(n)}(\mathfrak{Q})_{*}$ over $\lambda$ are simplicially homotopic.

The construction of $\check{C}^{(n)}$ directly implies that for any $n$-cube of groups $\mathfrak{F}, \check{C}^{(n)}(\mathfrak{F})_{*}$ is the diagonal of a bisimplicial group $F_{* *}$ induced by applying the ordinary Cech complex construction $\check{C}$ to the morphism of simplicial groups $\check{C}^{(n-1)}\left(\mathfrak{F}^{\overline{\{l\}}}\right)_{*} \rightarrow$ $\check{C}^{(n-1)}\left(\mathfrak{F}^{\{l\}}\right)_{*}$.

By assumption the extensions $\kappa$ and $\pi$ of the group morphism $\lambda$ induce a commutative diagram of simplicial groups

$$
\begin{array}{ccc}
\check{C}^{(n-1)}\left(\mathfrak{F}^{\overline{\{l\}}}\right)_{*} & \underset{\widetilde{\pi^{\prime}}}{\stackrel{\widetilde{\kappa^{\prime}}}{\rightrightarrows}} \check{C}^{(n-1)}\left(\mathfrak{Q}^{\overline{\{l\}}}\right)_{*} \\
\downarrow & \downarrow \\
\check{C}^{(n-1)}\left(\mathfrak{F}^{\{l\}}\right)_{*} & \underset{\kappa^{\prime \prime}}{\stackrel{\pi^{\prime \prime}}{\rightrightarrows}} & \check{C}^{(n-1)}\left(\mathfrak{Q}^{\{l\}}\right)_{*}
\end{array},
$$

where $\widetilde{\kappa^{\prime \prime}}=\widetilde{\pi^{\prime \prime}}$, which implies there are morphisms of simplicial objects of simplicial groups

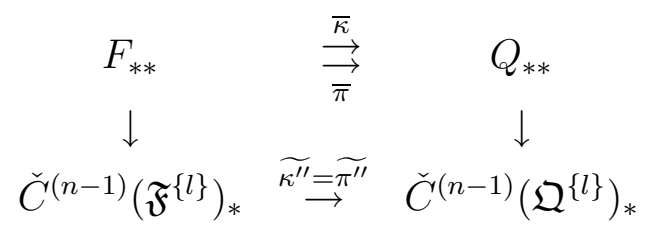

over the morphism of simplicial groups $\widetilde{\kappa^{\prime \prime}}=\widetilde{\pi^{\prime \prime}}$.

The following lemmas will be needed.

Lemma 17. Let $G_{* *}, H_{* *}$ be bisimplicial groups and $\alpha, \beta: G_{* *} \rightarrow H_{* *}$ morphisms of bisimplicial groups. Let there exist a vertical (horizontal) simplicial homotopy $h^{v}\left(h^{h}\right)$ between the induced morphisms of simplicial groups $\alpha_{m}, \beta_{m}: G_{m *} \rightarrow H_{m *}\left(G_{* m} \rightarrow\right.$ $H_{* m}$ ) for all $m \geq 0$, such that the following conditions hold:

$$
d_{j}^{h} h_{i}^{v}=h_{i}^{v} d_{j}^{h} \quad\left(d_{j}^{v} h_{i}^{h}=h_{i}^{h} d_{j}^{v}\right) .
$$

Then the induced morphisms of simplicial groups $\widetilde{\alpha}, \widetilde{\beta}: \Delta G_{*} \rightarrow \Delta H_{*}$ are simplicially homotopic, $\widetilde{\alpha} \simeq \widetilde{\beta}$, where $\Delta G_{*}$ and $\Delta H_{*}$ are the diagonal simplicial groups of $G_{* *}$ and $H_{* *}$ respectively.

Proof. We can construct the required homotopy in the following way: $h_{i}^{\prime}=h_{i}^{v} s_{i}^{h}: G_{n n} \rightarrow H_{n+1, n+1}, 0 \leq i \leq n$.

Now we have to check the standard identities for simplicial homotopy. In fact,

$$
\begin{aligned}
d_{0}^{v} d_{0}^{h} h_{0}^{v} s_{0}^{h} & =d_{0}^{v} h_{0}^{v} d_{0}^{h} s_{0}^{h}=d_{0}^{v} h_{0}^{v}=\alpha_{n n}, \\
d_{n+1}^{v} d_{n+1}^{h} h_{n}^{v} s_{n}^{h} & =d_{n+1}^{v} h_{n}^{v} d_{n+1}^{h} s_{n}^{h}=d_{n+1}^{v} h_{n}^{v}=\beta_{n n}, \\
d_{i}^{v} d_{i}^{h} h_{j}^{v} s_{j}^{h} & =d_{i}^{v} h_{j}^{v} d_{i}^{h} s_{j}^{h}=\left\{\begin{array}{c}
h_{j-1}^{v} d_{i}^{v} s_{j-1}^{h} d_{i}^{h}=h_{j-1}^{v} s_{j-1}^{h} d_{i}^{v} d_{i}^{h}, i<j \\
h_{j}^{v} d_{i-1}^{v} s_{j}^{h} d_{i-1}^{h}=h_{j}^{v} s_{j}^{h} d_{i-1}^{v} d_{i-1}^{h}, i>j+1
\end{array},\right. \\
d_{j+1}^{v} d_{j+1}^{h} h_{j+1}^{v} s_{j+1}^{h} & =d_{j+1}^{v} h_{j+1}^{v} d_{j+1}^{h} s_{j+1}^{h}=d_{j+1}^{v} h_{j+1}^{v}=d_{j+1}^{v} h_{j}^{v} d_{j+1}^{h} s_{j}^{h}=d_{j+1}^{v} d_{j+1}^{h} h_{j}^{v} s_{j}^{h} .
\end{aligned}
$$


Lemma 18. Let $\left(\check{C}(\alpha)_{*}, \alpha, G\right),\left(\check{C}(\beta)_{*}, \beta, H\right)$ be augmented $\check{C}$ ech complexes and $f, g: \check{C}(\alpha)_{*} \rightarrow \check{C}(\beta)_{*}$ morphisms of simplicial groups over a given group morphism $\lambda: G \rightarrow H$. Then $f$ and $g$ are simplicially homotopic, $f \simeq g$.

Proof. We only construct the simplicial homotopy and leave the checking of the corresponding identities to the reader. We define $h_{i}: \check{C}(\alpha)_{n} \rightarrow \check{C}(\beta)_{n+1}, 0 \leq i \leq n$, by

$$
h_{i}\left(x_{0}, \ldots, x_{n}\right)=\left(g\left(x_{0}\right), \ldots, g\left(x_{i}\right), f\left(x_{i}\right), \ldots, f\left(x_{n}\right)\right)
$$

for all $\left(x_{0}, \ldots, x_{n}\right) \in \check{C}(\alpha)_{n}$.

Returning to the main proof, using Lemma 18, it is easy to see that there exists a vertical homotopy $h^{v}$ between the induced morphisms of simplicial groups $\overline{\kappa_{m}}, \overline{\pi_{m}}$ : $F_{m *} \rightarrow Q_{m *}$ for all $m \geq 0$, such that $d_{j}^{h} h_{i}^{v}=h_{i}^{v} d_{j}^{h}$. Applying Lemma 17 there is a simplicial homotopy between the morphisms of simplicial groups $\widetilde{\kappa}, \widetilde{\pi}: \check{C}^{(n)}(\mathfrak{F})_{*} \rightarrow$ $\check{C}^{(n)}(\mathfrak{Q})_{*}$.

Now we return to the general case, showing for any two extensions $\kappa, \pi: \mathfrak{F} \rightarrow \mathfrak{Q}$ of a group morphism $\lambda: G \rightarrow H$ the existence of extensions $\kappa_{1}, \ldots, \kappa_{n-1}: \mathfrak{F} \rightarrow \mathfrak{Q}$ of $\lambda$ such that $\widetilde{\kappa} \simeq \widetilde{\kappa_{1}}, \widetilde{\kappa_{1}} \simeq \widetilde{\kappa_{2}}, \ldots, \widetilde{\kappa_{n-2}} \simeq \widetilde{\kappa_{n-1}}, \widetilde{\kappa_{n-1}} \simeq \widetilde{\pi}$ which, of course, implies that $\widetilde{\kappa} \simeq \widetilde{\pi}$. In fact, we can construct an extension $\kappa_{1}: \mathfrak{F} \rightarrow \mathfrak{Q}$ in the following way: let $\kappa_{1}^{\{1\}}=\kappa^{\{1\}}$ and $\kappa_{1}^{\langle n\rangle \backslash\{1\}}=\pi^{\langle n\rangle \backslash\{1\}}$. We complete the construction of $\kappa_{1}$ using the technique above and the facts that $\mathfrak{F}$ is a free and $\mathfrak{Q}$ is an exact $n$-presentation of the groups $G$ and $H$ respectively.

We construct an extension $\kappa_{i}$ for all $2 \leq i \leq n-1$ as follows: let $\kappa_{i}^{\{i\}}=\kappa_{i-1}^{\{i\}}$ and $\kappa_{i}^{\langle n\rangle \backslash\langle i\rangle}=\pi^{\langle n\rangle \backslash\langle i\rangle}$. We complete again the constructing of $\kappa_{i}$ using the above technique and the facts that $\mathfrak{F}$ is free and $\mathfrak{Q}$ is exact.

The construction of $\kappa_{i}, 1 \leq i \leq n-1$, and our already proved particular case imply that $\widetilde{\kappa} \simeq \widetilde{\kappa_{1}}, \widetilde{\kappa_{1}} \simeq \widetilde{\kappa_{2}}, \ldots, \widetilde{\kappa_{n-2}} \simeq \widetilde{\kappa_{n-1}}, \widetilde{\kappa_{n-1}} \simeq \widetilde{\pi}$.

Using this comparison theorem we make the following

Definition. Let $T: \mathfrak{G} r \rightarrow \mathfrak{G} r$ be a covariant functor. Define $i$-th $n$-fold $\check{C}$ ech derived functor $\mathcal{L}_{i}^{n-\text { fold }} T: \mathfrak{G} r \rightarrow \mathfrak{G} r, i \geq 0$, of the functor $T$ by choosing for each $G$ in $\mathfrak{G} r$, a free exact $n$-presentation $\mathfrak{F}$ and setting

$$
\mathcal{L}_{i}^{n-\text { fold }} T(G)=\pi_{i}\left(T \check{C}^{(n)}(\mathfrak{F})_{*}\right),
$$

where $\left(\check{C}^{(n)}(\mathfrak{F})_{*}, \alpha, G\right)$ is the $n$-fold Čech resolution of the group $G$ for the free exact $n$-presentation $\mathfrak{F}$ of $G$.

The $n$-fold Čech complexes and hence the $n$-fold Čech derived functors are closely related to the diagonal of the $n$-simplicial multinerve of crossed $n$-cubes of groups . In particular, we have the following

Lemma 19. Let $\mathfrak{F}$ be an n-presentation of a group $G$. There is an isomorphism of simplicial groups

$$
\check{C}^{(n)}(\mathfrak{F})_{*} \cong E^{(n)}(\mathcal{M})_{*}
$$


where $\mathcal{M}$ is the inclusion crossed $n$-cube of groups given by the normal $(n+1)$-ad of groups $\left(F ; R_{1}, \ldots, R_{n}\right)$ induced by $\mathfrak{F}$.

Proof. For $n=1$, is done in Lemma 1 and hence for general $n$, both constructions gives an isomorphism of $n$-simplicial groups. Applying the diagonal clearly gives the result.

The following theorem gives the $n$-th $n$-fold Čech derived functor of the functor $Z_{k}: \mathfrak{G} r \rightarrow \mathfrak{G} r, k \geq 2$.

Theorem 20. Let $G$ be a group and $k \geq 2$. Then there is an isomorphism

$$
\mathcal{L}_{n}^{n-\text { fold }} Z_{k}(G) \cong \frac{\bigcap_{i \in\langle n\rangle} R_{i} \cap \Gamma_{k}(F)}{D_{k}\left(F ; R_{1}, \ldots, R_{n}\right)}, \quad n \geq 1,
$$

where $\left(F ; R_{1}, \ldots, R_{n}\right)$ is the normal $(n+1)$-ad of groups induced by some free exact $n$-presentation $\mathfrak{F}$ of the group $G$.

Proof. By Definition and Lemma $19, \mathcal{L}_{n}^{n-\text { fold }} Z_{k}(G) \cong \pi_{n}\left(Z_{k} E^{(n)}(\mathcal{M})\right)_{*}$, where $\mathcal{M}$ is the inclusion crossed $n$-cube of groups given by the normal $(n+1)$-ad of groups $\left(F ; R_{1}, \ldots, R_{n}\right)$. Hence using Proposition 12 one has an isomorphism $\mathcal{L}_{n}^{n-\text { fold }} Z_{k}(G) \cong$ $\pi_{n}\left(E^{(n)} \mathcal{B}_{k}(\mathcal{M})_{*}\right)$. Then, by Proposition 14,

$$
\mathcal{L}_{n}^{n-\text { fold }} Z_{k}(G) \cong \bigcap_{l \in\langle n\rangle} \operatorname{Ker}\left(\mathcal{B}_{k}(\mathcal{M})_{\langle n\rangle} \stackrel{\widetilde{\mu_{l,\langle n\rangle}}}{\longrightarrow} \mathcal{B}_{k}(\mathcal{M})_{\langle n\rangle \backslash\{l\}}\right) .
$$

Now we set up the inductive hypothesis. Let $n=1$, then

$$
\mathcal{L}_{1}^{1-\text { fold }} Z_{k}(G) \cong \operatorname{Ker}\left(\frac{R_{1}}{D_{k}\left(F ; R_{1}\right)} \longrightarrow \frac{F}{\Gamma_{k}(F)}\right)=\frac{R_{1} \cap \Gamma_{k}(F)}{D_{k}\left(F ; R_{1}\right)} .
$$

Proceeding by induction, we suppose that the result is true for $n-1$ and we will prove it for $n$.

Let us consider $l \in\langle n\rangle$. It is easy to check that $\mathfrak{F}^{\overline{\{l\}}}$ is a free exact $(n-1)$ presentation of the free group $\mathfrak{F}_{\langle n\rangle \backslash\{l\}}$. Here we have to use the fact that if $G$ is a free group, then $\mathcal{L}_{i}^{n-\text { fold }} T(G)=0, i>0$ and $\mathcal{L}_{0}^{n-\text { fold }} T(G) \cong T(G)$ for any functor $T: \mathfrak{G} r \rightarrow \mathfrak{G} r$. Thus, because of our inductive hypothesis,

$$
\mathcal{L}_{n-1}^{(n-1)-\text { fold }} Z_{k}\left(\mathfrak{F}_{\langle n\rangle \backslash\{l\}}\right) \cong \frac{\bigcap_{i \in\langle n\rangle \backslash\{l\}} R_{i} \cap \Gamma_{k}(F)}{D_{k}\left(F ; R_{1}, \ldots, R_{l-1}, R_{l+1}, \ldots, R_{n}\right)}=0 .
$$

Now from (3) and (4) one can easily deduce that there is the isomorphism

$$
\mathcal{L}_{n}^{n-\text { fold }} Z_{k}(G) \cong \frac{\bigcap_{i \in\langle n\rangle} R_{i} \cap \Gamma_{k}(F)}{D_{k}\left(F ; R_{1}, \ldots, R_{n}\right)} .
$$

Now we are ready to improve on Theorem BE, and moreover to express by generalised Hopf formulae not only the non-abelian derived functors of the functor $Z_{2}$, but also the derived functors of the functors $Z_{k}, k \geq 2$. 
Theorem 21. Let $G$ be a group, $\mathfrak{F}$ be a free exact $n$-presentation of $G$ and $k \geq 2$. Then

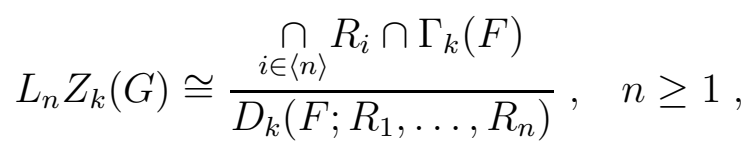

where $\left(F ; R_{1}, \ldots, R_{n}\right)$ is the normal $(n+1)$-ad of groups induced by $\mathfrak{F}$.

Proof. This directly follows from Corollary 10, Proposition 15(ii) and Theorem 20.

Remark. One can prove an analogous result in a more general context of Theorem $\mathrm{BE}$ and Corollary 4. In particular, for a given group $G$ and an exact $n$-presentation $\mathfrak{F}$ of $G$ such that

$$
L_{1} Z_{k}\left(\mathfrak{F}_{\emptyset}\right)=0, \quad L_{r} Z_{k}\left(\mathfrak{F}_{A}\right)=0 \text { for } r=|A|, r=|A|+1
$$

with $A$ a non-empty proper subset of $\langle n\rangle$, there is an isomorphism

$$
L_{n} Z_{k}(G) \cong \frac{\cap_{i \in\langle n\rangle} R_{i} \cap \Gamma_{k}(F)}{D_{k}\left(F ; R_{1}, \ldots, R_{n}\right)} \text { for } n \geq 1,
$$

where $\left(F ; R_{1}, \ldots, R_{n}\right)$ is the normal $(n+1)$-ad of groups induced by $\mathfrak{F}$.

Now we concentrate our attention on the computation of 2 -fold Čech derived functors of the functor $Z_{k}: \mathfrak{G} r \rightarrow \mathfrak{G} r, k \geq 2$. Using the fact that $Z_{k}, k \geq 2$ is a right exact functor one easily shows that $\mathcal{L}_{0}^{2}$-fold $Z_{k} \cong Z_{k}$. Moreover, by Proposition 12, Proposition 14 and Lemma $19, \mathcal{L}_{i}^{2 \text {-fold }} Z_{k}=0$ for $i \geq 3$. To take into account Theorem 20, it only remains to compute the first 2-fold Čech derived functor of the functor $Z_{k}$.

Lemma 22. (cf. Conduché [7] and also, [21]) Let

$$
\mathcal{M}=\left\{\begin{array}{rll}
L & \stackrel{\lambda}{\rightarrow} & M \\
\lambda^{\prime} \downarrow & & \downarrow \mu \\
N & \stackrel{\nu}{\rightarrow} & P
\end{array}\right\}
$$

be a crossed square. Then

$$
\begin{aligned}
& H_{0}\left(C_{*}(\mathcal{M})\right)=P / \operatorname{Im} \mu \operatorname{Im} \nu, \\
& H_{1}\left(C_{*}(\mathcal{M})\right) \cong M \times_{P} N / \operatorname{Im} \kappa, \\
& H_{2}\left(C_{*}(\mathcal{M})\right)=\operatorname{Ker} \lambda \cap \operatorname{Ker} \lambda^{\prime},
\end{aligned}
$$

where $C_{*}(\mathcal{M})$ is the mapping cone complex of groups

$$
L \stackrel{\alpha}{\rightarrow} M \rtimes N \stackrel{\beta}{\rightarrow} P
$$

with $\alpha(l)=\left(\lambda(l)^{-1}, \lambda^{\prime}(l)\right), \beta(m, n)=\mu(m) \nu(n)$ for all $l \in L,(m, n) \in M \rtimes N$ and $\kappa$ is the natural homomorphism from $L$ to $M \times_{P} N$. 
Proof. We only prove that $H_{1}\left(C_{*}(\mathcal{M})\right) \cong M \times_{P} N / \operatorname{Im} \kappa$. It is easy to check that $f: \operatorname{Ker} \beta \rightarrow M \times{ }_{P} N$, given by $f(m, n)=\left(m^{-1}, n\right)$ for all $(m, n) \in \operatorname{Ker} \beta$, is an isomorphism and $\operatorname{Im} f \alpha=\operatorname{Im} \kappa$. The other results are as easy as this part to check.

Proposition 23. For a given group $G$ and $k \geq 2$ there are isomorphisms of groups

$$
L_{1} Z_{k}(G) \cong \mathcal{L}_{1}^{2-\text { fold }} Z_{k}(G) \cong \frac{R_{1} \Gamma_{k}(F) \cap R_{2} \Gamma_{k}(F)}{\left(R_{1} \cap R_{2}\right) \Gamma_{k}(F)},
$$

where $\left(F ; R_{1}, R_{2}\right)$ is a normal 3 -ad of groups induced by some free exact 2-presentation $\mathfrak{F}$ of $G$.

Proof. Begin with the first isomorphism. By the construction, $\check{C}^{(2)}(\mathfrak{F})_{*}$ is the diagonal of a bisimplicial group $F_{* *}$ induced by applying the ordinary Cech complex construction $\check{C}$ to the morphism of Čech complexes $\check{C}\left(\mathfrak{F}^{\overline{11\}}}\right)_{*} \rightarrow \check{C}\left(\mathfrak{F}^{\{1\}}\right)_{*}$. Now applying the right exact functor $Z_{k}$ dimension-wise, denote the resulted bisimplicial group $Z_{k}\left(F_{* *}\right)$. By [27] there is a spectral sequence

$$
E_{p q}^{2} \Longrightarrow \mathcal{L}_{p+q}^{2-\text { fold }} Z_{k}(G)
$$

where $E_{0 q}^{2}=0, q>0$ and $E_{p 0}^{2} \cong \mathcal{L}_{p}^{1-\text { fold }} Z_{k}(G), p \geq 0$. Hence there is an isomorphism $\mathcal{L}_{1}^{2-\text { fold }} Z_{k}(G) \stackrel{\cong}{\rightrightarrows} \mathcal{L}_{1}^{1-\text { fold }} Z_{k}(G)$. Now the required isomorphism follows from [26], (see also [12]).

Again use of Proposition 12, Proposition 14 and Lemma 19 implies that

$$
\mathcal{L}_{1}^{2-\text { fold }} Z_{k}(G) \cong H_{1}\left(C_{*}\left(\mathcal{B}_{k}(\mathcal{M})\right)\right.
$$

where $\mathcal{M}$ is the inclusion crossed square induced by the normal 3-ad of groups $\left(F ; R_{1}, R_{2}\right)$. Since the homomorphisms

$$
\widetilde{\mu_{1}}: \mathcal{B}_{k}(\mathcal{M})_{\{1\}} \rightarrow \mathcal{B}_{k}(\mathcal{M})_{\emptyset}
$$

and

$$
\widetilde{\mu_{2}}: \mathcal{B}_{k}(\mathcal{M})_{\{2\}} \rightarrow \mathcal{B}_{k}(\mathcal{M})_{\emptyset}
$$

are injections, using Lemma 22 implies the second isomorphism.

Note that for group-abelianization functor $A b=Z_{2}$ we have the following new interpretation of the second integral group homology

$$
H_{2}(G) \cong \mathcal{L}_{1}^{2-\text { fold }} A b(G) \cong \frac{R_{1}[F, F] \cap R_{2}[F, F]}{\left(R_{1} \cap R_{2}\right)[F, F]} .
$$

It is an interesting problem to investigate and to compute the functors $\mathcal{L}_{i}^{n \text {-fold }} Z_{k}$ for $0<i<n, n \geq 3$. 


\section{Hopf type FORMulas in Algebraic $K$-THEORY}

Let us recall the well-known definition of $\overleftarrow{j}_{j}^{\lim }(1)$, the first derived functor of the functor $\underset{\longleftarrow_{j}}{\lim }$ (inverse limit in the category of groups)(see e.g. [12]). Let $\left\{A_{j}, p_{j+1}^{j}\right\}_{j}$ be a countable inverse system of groups, then

$$
\overleftarrow{\lim }_{j}^{(1)}\left\{A_{j}, p_{j+1}^{j}\right\}=\prod_{j} A_{j} / \sim,
$$

where $\sim$ is an equivalence relation on the set $\prod_{j} A_{j}$ defined as follows: $\left\{a_{j}\right\} \sim\left\{a_{j}^{\prime}\right\}$ if there exists $\left\{h_{j}\right\}$ such that $\left\{h_{j}\right\}\left\{a_{j}\right\}\left\{p_{j+1}^{j}\left(h_{j+1}^{-1}\right)\right\}=\left\{a_{j}^{\prime}\right\}$.

Let $\left\{G_{*}^{j}, \psi_{j+1}^{j}\right\}_{j}$ be a countable inverse system of pseudosimplicial groups $G_{*}^{j}$ with $\psi_{j+1}^{j}: G_{*}^{j+1} \rightarrow G_{*}^{j}$ a fibration for all $j \geq 1$. Let $G_{*}=\underset{\longleftarrow}{\lim }\left\{G_{*}^{j}, \psi_{j+1}^{j}\right\}$.

Theorem 24. [12] There is a short exact sequence of groups

$$
0 \longrightarrow \overleftarrow{j}^{\lim }{ }^{(1)} \pi_{n+1}\left(G_{*}^{j}\right) \longrightarrow \pi_{n}\left(G_{*}\right) \longrightarrow \underset{\longleftarrow_{j}}{\lim } \pi_{n}\left(G_{*}^{j}\right) \longrightarrow 0
$$

for all $n \geq 0$.

Let us define the functor $Z_{\infty}: \mathfrak{G} r \rightarrow \mathfrak{G} r$ as follows: for a given group $G$, $Z_{\infty}(G)={\underset{\leftarrow}{j}}_{\lim } Z_{j}(G)$; for a given group homomorphism $\lambda: G \rightarrow H, Z_{\infty}(\lambda)$ is the group homomorphism induced by the $Z_{j}(\lambda)$.

It is known from [14] (see also [12]) that the non-abelian left derived functors $L_{*}^{\mathcal{P}} Z_{\infty}$ of the functor $Z_{\infty}: \mathfrak{G} r \rightarrow \mathfrak{G} r$ are isomorphic to Quillen's $K$-functors. Thus using Theorem 24 we deduce that there is a short exact sequence of abelian groups

$0 \longrightarrow \overleftarrow{j}^{\lim }{ }^{(1)} \pi_{n+1}\left(F_{*}^{j}(G L(\Re))\right) \longrightarrow K_{n+1}(\Re) \longrightarrow \overleftarrow{j}_{\lim _{j}}^{\lim } \pi_{n}\left(F_{*}^{j}(G L(\Re))\right) \longrightarrow 0, \quad n \geq 0$

where $F_{*}(G L(\Re)) \rightarrow G L(\Re)$ is a free pseudosimplicial resolution of the group $G L(\mathfrak{R})$ and $F_{*}^{j}(G L(\mathfrak{R}))=Z_{j}\left(F_{*}(G L(\Re))\right)$.

Now according to Corollary 10 we obtain the following

Theorem 25. Let $\mathfrak{R}$ be a ring with unit and $\left(F_{*}, d_{0}^{0}, G L(\Re)\right)$ be a free pseudosimplicial resolution of the general linear group $G L(\mathfrak{R})$. Then there is an exact sequence 
of abelian groups

$$
\begin{aligned}
0 \longrightarrow & \overleftarrow{\lim }_{j}^{(1)}\left(\frac{\left(\cap_{i \in\langle n+1\rangle} \operatorname{Ker} d_{i-1}^{n}\right) \cap \Gamma_{j}\left(F_{n}\right)}{D_{j}\left(F_{n} ; \operatorname{Ker} d_{0}^{n}, \ldots, \operatorname{Ker} d_{n}^{n}\right)}\right) \longrightarrow K_{n+1}(\Re) \longrightarrow \\
\longrightarrow & \underset{\lim _{j}}{\longleftarrow}\left(\frac{\left(\cap_{i \in\langle n\rangle} \operatorname{Ker} d_{i-1}^{n-1}\right) \cap \Gamma_{j}\left(F_{n-1}\right)}{D_{j}\left(F_{n-1} ; \operatorname{Ker} d_{0}^{n-1}, \ldots, \operatorname{Ker} d_{n-1}^{n-1}\right)}\right) \longrightarrow 0
\end{aligned}
$$

for $n \geq 1$.

Note that using Theorem 21 and its Remark, one can express $K_{n+1}(\mathfrak{R})$ in data coming from exact $(n+1)$ and $n$-presentations of the group $G L(\Re)$. We hope to return to a more detailed analysis of this in future work.

\section{Acknowledgements}

The second author would like to thank the Royal Society, and University of Wales Bangor for their hospitality at the 'initialisation' of this paper. The authors were partially supported by INTAS grant 00566 , and in addition the third author by INTAS 971 - 31961. The first and the second authors were also supported by INTAS Georgia grant No 213, whilst the second author was supported by FNRS grant 7GEPJ065513.01.

\section{REFERENCES}

[1] M. Barr and J. Beck, Homology and Standard Constructions, Lecture Notes in Mathematics, Vol.80, Springer-Verlag, Berlin/New York (1969), 245-335.

[2] H.-J. Baues and D. Conduché, The Central Series for Peiffer Commutators in Groups with Operators, J.Algebra Vol.133, No.1 (1990), 1-34.

[3] R. Brown and G.J. Ellis, Hopf formulae for the higher homology of a group, Bull. London Math. Soc. 20 (1988), 124-128.

[4] R. Brown and J.-L. Loday, Van Kampen theorems for diagrams of spaces, Topology 26 (1987), 311-335.

[5] R. Brown and J.-L. Loday, Homotopical excision, and Hurewicz theorems, for $n$-cubes of spaces, Proc. London Math. Soc. (3) 54 (1987), 176-192.

[6] D. Conduché, Modules Croisés Généralisés de Longueur 2, J. Pure Appl. Algebra, 34, (1984), 155-178.

[7] D. Conduché, Letter to R. Brown and J.-L. Loday, 1984.

[8] D. Conduché, Simplicial crossed modules and mapping cones, preprint, Université de Rennes I, 2002.

[9] B. Conrad, Crossed $n$-fold extensions of groups, $n$-fold extensions of modules, and higher multipliers, J. Pure Appl. Algebra, 36 (1985), 225-235.

[10] G.J. Ellis, Relative derived functors and the homology of groups, Cahiers de Top. et Géom. Diff. cat. XXXI-2 (1990), 121-135.

[11] G.L. Ellis and R. Steiner, Higher dimensional crossed modules and the homotopy groups of $(n+1)-a d s$, J. Pure Appl. Algebra 46 (1987), 117-136. 
[12] H. Inassaridze, Non-abelian homological algebra and its applications, Kluwer Academic Publishers, Amsterdam, 1997.

[13] N. Inassaridze and E. Khmaladze, More about homological properties of precrossed modules, Homology, Homotopy and Applications, vol.2, No.7 (2000), 105-114.

[14] F. Keune, Derived functors and algebraic K-theory, Lecture Notes in Math., Springer-Verlag 341 (1973), 158-168.

[15] F. Keune, Multirelative $K$-theory and axioms for the $K$-theory of rings, K-theory Preprint Archives 137 (1996) (http://www.math.uiuc.edu/K-theory/).

[16] M.Ladra and A.R.-Grandjeán, Crossed modules and homology, J.Pure Appl. Algebra 95 (1994), 41-55.

[17] J.-L. Loday, Spaces with finitely many non-trivial homotopy groups, J. Pure Appl. Algebra 24 (1982), 179-202.

[18] J.-L. Loday, Dialgebras, in "Dialgebras and related operads", Lecture Notes in Math., Springer-Verlag 1763 (2001), 7-66.

[19] A. Mutlu and T. Porter, Iterated Peiffer pairings in the Moore complex of a simplicial group, Applied Categorical Structures 9 (2001) 111-130.

[20] A. Mutlu and T. Porter, Applications of Peiffer pairings in the Moore complex of a simplicial group, Theory and Applications of Categories, 4, No.7, (1998), 148-173.

[21] A. Mutlu and T. Porter, Crossed squares and 2-crossed modules, preprint, Bangor, 2002.

[22] T. Pirashvili, On non-abelian derived functors, Proc. A.Razmadze Math. Inst., Georgian Acad. Sci., 42 (1979), 91-104 (in Russian).

[23] T. Pirashvili, Spectral sequence for epimorphism. I, Proc. Math. Inst. Tbilisi 70 (1982), 69-91 (in Russian).

[24] T. Pirashvili, Simplicial degrees of functors, Math. Proc. Camb. Phil. Soc. 126 (1999), 45-62.

[25] T. Porter, n-types of simplicial groups and crossed n-cubes, Topology, Vol.32, No.1 (1993), 5-24.

[26] T. Pirashvili, On non-abelian derived functors, Proc. A.Razmadze Math. Inst., Georgian Acad. Sci., 42 (1979), 91-104 (in Russian).

[27] D. Quillen, Spectral sequences of a double semi-simplicial group, Topology 5 (1966), 155-157.

[28] A. Rodicio, Presentaciones libres y $H_{2 n}(G)$, Publ. Mat. Univ. Aut. Barcelona 30 (1986), $77-82$.

[29] S. Shatz, Profinite groups, arithmetic, and geometry, Princeton Univ. Press, 1972.

[30] R. Stöhr, A generalized Hopf formula for higher homology groups, Comment. Math. Helvetici 64 (1989), 187-199. 